\title{
ANÁLISIS FITOLÍTICO DE LA FORMACIÓN TEZANOS PINTO (PLEISTOCENO TARDÍO-HOLOCENO TEMPRANO) EN EL SECTOR SUDOESTE DE LA PROVINCIA DE ENTRE RÍOS (ARGENTINA)
}

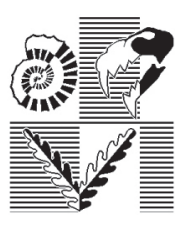

\author{
GEORGINA ERRA ${ }^{1,4}$, ALEJANDRO F. ZUCOL ${ }^{2,4}$, DANIELA M. KRÖHLING ${ }^{3,4}$ Y CARLOS A. GONZÁLEZ ${ }^{2,4}$
}

${ }^{1}$ Centro de Ecología Aplicada del Litoral, Área de Paleontología, CONICET, Ruta 5, km 2.5, CC.128, 3400 Corrientes, Argentina - Cátedra de Paleontología 2, Facultad de Ciencias Naturales y Museo, Universidad Nacional de La Plata, La Plata, Argentina. georginaerra@yahoo.com.ar

${ }^{2}$ Laboratorio de Paleobotánica, Centro de Investigaciones Científicas y Transferencia de Tecnología a la Producción (CICYTTP-CONICET), Dr. Materi y Espańa, E3105BWA Diamante, Entre Ríos, Argentina - Facultad de Ciencia y Tecnología, Universidad Autónoma de Entre Ríos, Subsede Diamante, Entre Ríos, Argentina. cidzucol@infoaire.com.ar;adr_1103@hotmail.com

${ }^{3}$ Facultad de Ingeniería y Ciencias Hídricas, Universidad Nacional del Litoral, CC217, 3000 Santa Fe, Argentina.dkrohli@gmail.com

${ }^{4}$ Consejo Nacional de Investigaciones Científicas y Técnicas (CONICET)

Resumen. Los depósitos de loess son el principal componente de las secuencias sedimentarias del Cuaternario en la llanura pampeana (América del Sur). El loess generado durante el Último Máximo Glacial, representa una unidad depositacional del Sistema Eólico Pampeano, que evidencia la extensión de condiciones semiáridas hacia el noreste de la región. La Formación Tezanos Pinto es la unidad loéssica típica del Pleistoceno tardío-Holoceno temprano en el noreste de la región pampeana. Los avances en el conocimiento micropaleobotánico de esta formación geológica son presentados en este trabajo, especialmente los obtenidos en la zona sudoeste de la provincia de Entre Ríos. Las asociaciones fitolíticas analizadas muestran una alta homogeneidad composicional, con algunas variaciones que permitieron la diferenciación cuantitativa a través de los diferentes perfiles sedimentarios. La presencia de fitolitos de gramíneas en asociación con los de palmeras y los elementos asignables a podostemáceas y ciperáceas han permitido realizar una caracterización de estas secuencias. Las asociaciones fitolíticas descriptas, como se observó previamente en la zona noroeste del área cubierta por el loess en Entre Ríos, mostraron una gran abundancia de gramíneas megatérmicas, con una mayor cantidad de elementos xéricos en los niveles inferiores y elementos que denotan episodios de clima templado-cálido y buena disponibilidad hídrica, principalmente en las secciones media y superior. Esto está relacionado con la presencia de una estepa de clima templado cálido a templado, con condiciones xéricas principalmente en los niveles basales.

Palabras clave. Fitolitos. Loess. Pleistoceno-Holoceno. Entre Ríos. Argentina.

\begin{abstract}
PHYTOLITH ANALYSIS OF TEZANOS PINTO FORMATION (LATE PLEISTOCENE-HOLOCENE) IN THE SOUTHWESTERN REGION OF ENTRE RÍOS PROVINCE (ARGENTINA). Loess deposits are the main component of the Late Quaternary sequences of the Pampa plains (South America). The Last Glacial Maximum loess represents a depositional unit of the Pampean Aeolian System that evidences the expansion of semiarid conditions to the northeast of the region. The Tezanos Pinto Formation is the typical loessic unit of the late Pleistocene-early Holocene of the northeastern Pampa region. Advances in the knowledge of the micropaleobotanical content of this geologic formation are presented in this work, especially the obtained results of the Southweastern area of Entre Ríos Province. The analyzed phytolith assemblages show a high homogeneity with some variations that allowed their quantitative differentiation across the analyzed sedimentary profiles. The presence of grass phytoliths jointly with palm, podostemoid and ciperoid elements allowed to make this characterization. The described phytolith assemblages, as previously observed in the northwestern part of the area covered by the loess in Entre Ríos, show high abundance of megathermic grasses, with a greater presence of xeric elements at lower levels, and with elements denoting warm-temperate episodes with major moisture principally in their middle and top sections. This is linked to the presence of a warm-temperate to temperate steppe with xeric conditions in the basal levels.
\end{abstract}

Keywords. Phytoliths. Loess. Pleistocene-Holocene. Entre Ríos. Argentina

Los estudios fitolíticos, conjuntamente con el análisis de otros tipos de restos como microcarbones y moluscos, resultaron ser importantes fuentes de información en depósitos de tipo loéssicos (Behrensmeyer y Hook, 1992). La presencia de fitolitos en sedimentos cuaternarios de la región pampeana fue tempranamente descripta por Fren- guelli $(1930)$. Teruggi $(1955,1957)$ utilizó estos conocimientos para interpretar la vegetación graminosa presente durante la acumulación del material loéssico. Bertoldi de Pomar $(1970,1980)$ realizó un estudio completo de los fitolitos en distintos tipos de sedimentos en la provincia de Santa Fe, incluyendo depósitos eólicos finos. La presencia 
de asociaciones de microfósiles vinculados a sedimentos loéssicos de Argentina ha sido utilizada más recientemente en las provincias de San Luis, Buenos Aires y Entre Ríos de acuerdo a lo recopilado por Zucol et al. (2008 y referencias allí citadas).

Para el caso particular de los depósitos loéssicos pampeanos, los análisis fitolíticos se presentan como un recurso valioso para aportar información sobre las condiciones bajo las cuales se depositaron los sedimentos finos que los componen (Osterrieth et al., 2008; Gutiérrez et al., 2011), mientras que en el área del Litoral argentino la aplicación de estudios fitolíticos ha permitido conocer las características de los ambientes de depositación (Kröhling et al., 2010; Erra, 2010a; Erra et al., 2011).

La distribución del loess pampeano en un mapeo inicial llevado a cabo por Teruggi (1957) fue muy generalizada y abarcó también superficies cubiertas por depósitos loéssicos generados a partir del retrabajo de sedimentos eólicos finos e incluso arenas eólicas y abanicos aluviales. Trabajos de detalle posteriores precisaron los mapeos de algunas unidades loéssicas típicas de la llanura pampeana. Entre ellas, la Formación Tezanos Pinto (Iriondo, 1980; Kröhling et al., 2010), que es una de las unidades más representativas del loess primario depositado durante el Último Máximo Glacial (UMG) en la Pampa Norte (Iriondo y Kröhling, 1995; Kröhling, 1999). El sudoeste de Entre Ríos (Fig. 1) forma parte del borde noreste del área de depositación de dicha formación geológica, que se extiende además por el centrosur de la provincia de Santa Fé y el este de la provincia de Córdoba (Iriondo y Kröhling, 1995, 2007). El sudoeste de Entre Ríos constituye un área de especial interés para el estudio de la composición fitolítica del loess primario, ya que

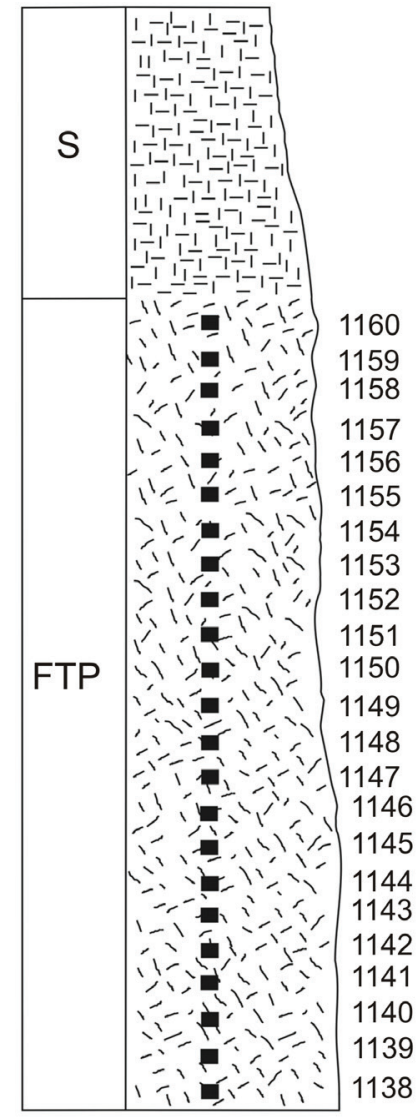

\section{PERFIL DIAMANTE NORTE}
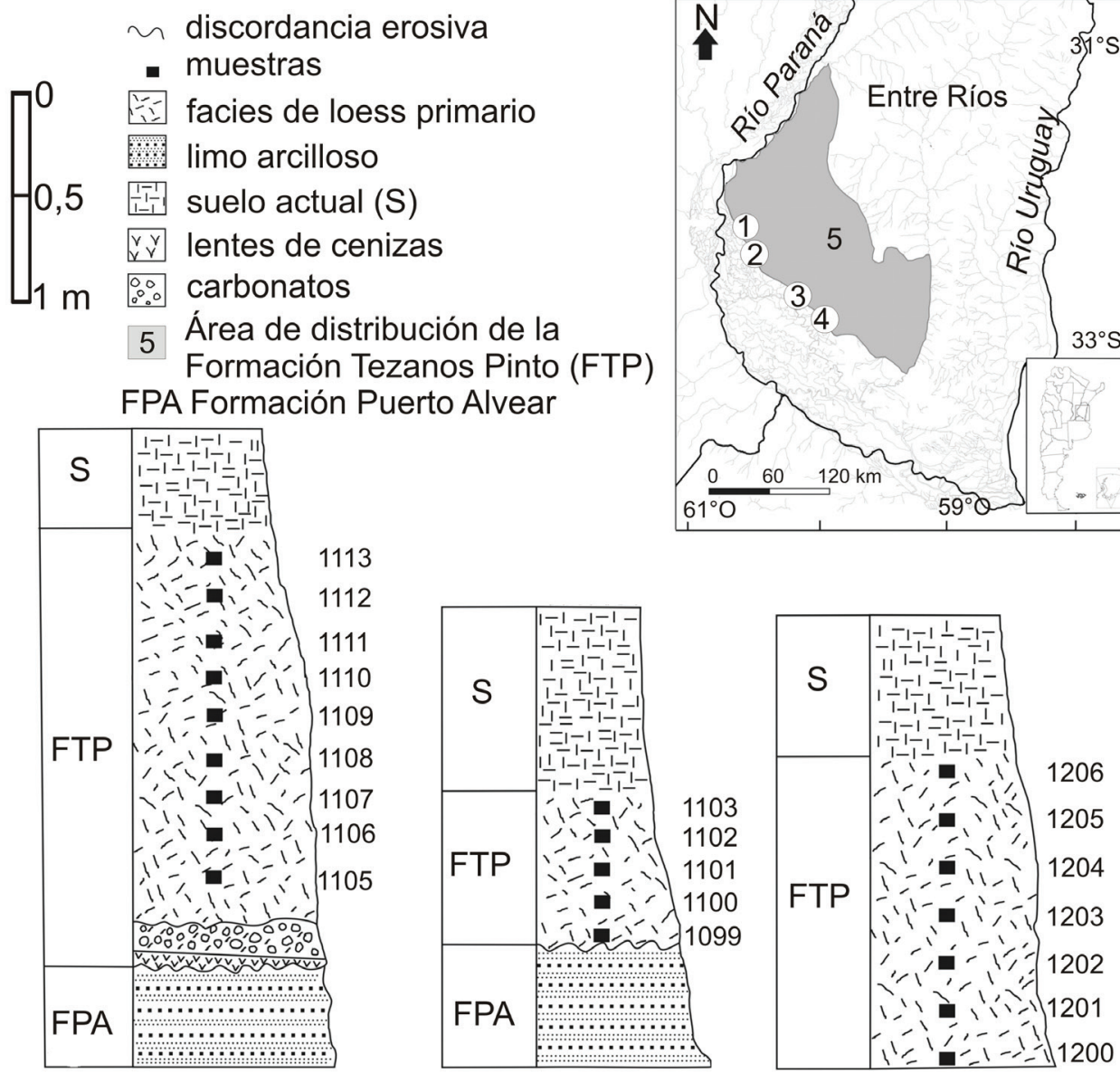

2) PERFIL GAUCHITO GIL 4 PERFIL VIALMAT 3 PERFIL LAS CUEVAS

Figura 1. Distribución de la Formación Tezanos Pinto en la provincia de Entre Ríos. Ubicación geográfica y litología de los perfiles muestreados 1. $32^{\circ} 03^{\prime} 59,6^{\prime \prime} \mathrm{S}-60^{\circ} 37^{\prime} 32,3^{\prime \prime} \mathrm{O} ; \mathbf{2}^{\circ} 32^{\circ} 18^{\prime} 51,5^{\prime \prime} \mathrm{S}-60^{\circ} 28^{\prime} 01,8^{\prime \prime} \mathrm{O} ; 3.32^{\circ} 36^{\prime} 37,3^{\prime \prime} \mathrm{S}-60^{\circ} 08^{\prime} 34,3^{\prime \prime} \mathrm{O} ; 4.32^{\circ} 46^{\prime} 05,2^{\prime \prime} \mathrm{S}-59^{\circ} 56^{\prime} 26,2^{\prime \prime} \mathrm{O}$. 
es la parte más distal del Sistema Eólico Pampeano (Iriondo y Kröhling, 1995).

La Formación Tezanos Pinto indica el avance del ambiente pampeano durante el UMG en la región, respecto de la influencia de los sistemas fluviales del Paraná y Uruguay, que dominaron durante el registro ambiental cuaternario de la Mesopotamia argentina (Kröhling, 2001).

En el sudoeste de Entre Ríos, la Formación Tezanos Pinto constituye la Unidad Geomorfológica "Colinas Loéssicas de Crespo" (Iriondo, 1998; fig. 1), cubriendo el paleorelieve en forma de manto. Las mayores potencias de la unidad se registran en el sector noroeste, correspondiente al área tipo de la formación (localidad tipo y perfiles accesorios). En los afloramientos de la barranca de la margen izquierda del Río Paraná, la unidad aflora con 2 a 4 m de espesor. Hacia el este se encuentra parcialmente erodada, aflorando en barrancas y cortes artificiales con espesores típicos de $1 \mathrm{a} 2 \mathrm{~m}$ en las partes altas del paisaje hasta muy cerca del Río Gualeguay. Hacia el sur la unidad disminuye su potencia.

Este trabajo es una continuación de los estudios que se están realizando en los depósitos de loess primario en Entre Ríos (Kröhling et al., 2006). En una primera etapa se avanzó en la investigación del área loéssica noroeste (Erra et al., 2011), siendo esta contribución una continuación de los estudios hacia el sector sur del área loéssica, próxima al valle fluvial del Paraná y del delta holoceno del Paraná. Las asociaciones fitolíticas previamente descriptas al norte de la presente área de estudio (Erra et al., 2011) se caracterizan por una elevada presencia de fitolitos graminoides y arecoides; donde los fitolitos de palmeras se presentan en forma subordinada a los demás morfotipos. Las composiciones fitolíticas resultaron con un alto grado de homogeneidad, en coincidencia con las características masivas del depósito, según criterios de campo. Un análisis de detalle permitió describir variaciones en general vinculadas a su distribución geográfica, como así también a las diferencias del propio material sedimentario. Así los perfiles periféricos han resultado los de mayor proporción de fitolitos vinculados con palmeras y ciperáceas, relacionado al ambiente de depositación del polvo eólico más cercano a elementos insulares, fluviales o cauces abandonados de los mismos. En contraste, en los perfiles centrales del área loéssica de Entre Ríos se encontraron mayores indicios de elementos graminoides, en algunos casos de características xéricas. Esta situación permite vincular a las asociaciones fitolíticas analizadas con un clima templado cálido de marcadas tendencias de aridez y variaciones que estarían relacionadas con ambientes locales diferenciados de la planicie general.

Los primeros datos relacionados con la variabilidad fitolítica del área loéssica del SO de Entre Ríos (Fig. 1), evidencian una diferenciación en dirección NO-SE, en coincidencia con la observada en la tendencia granulométrica de la unidad loéssica estudiada (Kröhling et al., 2010). Según el citado trabajo, la media granulométrica del loess a lo largo de la transecta SE-NO, de $170 \mathrm{~km}$ de longitud refleja un gradiente SE-NO en el tamaño de grano desde 19-23 $\mu \mathrm{m}$ (limo medio) en el margen SE a 9-14 $\mu \mathrm{m}$ (limo fino) en los perfiles del NO. El contenido de arena muy fina disminuye desde $16-20 \%$ en el SE a $4-14 \%$ en el NO; en cambio la fracción arcilla total aumenta desde $4-10 \%$ ( $<2 \%$ coloides) en el SE a 11-24\% (3-6\% coloides) en el área NO.

Dataciones por OSL (Optical Stimulated Luminescence) del perfil tipo de la Formación Tezanos Pinto, en la provincia de Entre Ríos ( $\left.31^{\circ} 53^{\prime} 20.7^{\prime \prime} \mathrm{S}-60^{\circ} 32^{\prime} 28.2^{\prime \prime} \mathrm{O}\right)$ indicaron edades de $32.640 \pm 2120$ ańos AP y $24.040 \pm 1570$ años $\mathrm{AP}$, sobre muestras de sedimentos tomadas a $3,5 \mathrm{~m}$ de profundidad respecto de la superficie del terreno (facies pa-

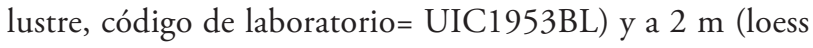
primario, código de laboratorio $=$ UIC1954BL), respectivamente. La fauna de moluscos preservada en el loess de la formación, es abundante pero con baja diversidad, y se encuentra caracterizada por Succinea meridionalis d'Orbigny 1842 (edad 14C de $27.650 \pm 25014 \mathrm{C}$ años AP), asociada con Biomphalaria peregrine d'Orbigny 1835 (24.390 \pm 170 ${ }^{14} \mathrm{C}$ ańos AP) (Kröhling et al., 2010).

Kröhling (1998) definió dos miembros en la Formación Tezanos Pinto en el centro-sur de la provincia de Santa Fe (al oeste del área de estudio de este trabajo), en base a la presencia de una discordancia intraformacional en los perfiles y en los resultados de dataciones TL (Thermoluminescence) y OSL. Dichos datos indican que el miembro inferior se depositó entre 36.000 y 16.000 ańos AP, mientras que el miembro superior se acumuló entre 14.000 y 8.000 años AP. En consecuencia, las dataciones obtenidas en el perfil tipo de la unidad litoestratigráfica y las características litológicas observadas en el SO de Entre Ríos, permiten deducir que en la región de estudio domina el miembro inferior de la Formación Tezanos Pinto (UMG, Pleistoceno Tardío).

\section{ÁREA DE ESTUDIO Y MARCO GEOLÓGICO}

De acuerdo con Kröhling et al. (2006), la Formación Tezanos Pinto en el sudoeste de Entre Ríos está típicamen- 
te representada por la facies eólica o loess primario. Este loess constituye taludes prácticamente verticales en la parte superior de la barranca del Río Paraná, en donde la formación descansa en discordancia erosiva sobre el Grupo Punta Gorda, con espesores de 2 a $3 \mathrm{~m}$. Es un depósito masivo, en general friable, permeable, de color marrón (7.5 YR 5/4); está atravesado por finos canalículos radiculares y poros muy finos tubulares, conteniendo localmente segregaciones de sesquióxidos de hierro y manganeso. El sedimento (compuesto entre un 60 a $80 \%$ del total por la fracción limo 64-4 $\mu \mathrm{m}$ ) presenta carbonatos segregados, formando concreciones esféricas de $1 \mathrm{~cm}$ de diámetro promedio, rizoconcreciones de disposición preferencial vertical y pseudomicelios. En el techo de esta formación se halla un suelo enterrado holoceno, moderadamente a bien desarrollado. El suelo fue truncado por erosión y se encuentra cubierto por un depósito loéssico (Formación San Guillermo), de 20 a $50 \mathrm{~cm}$ de espesor, de edad Holoceno tardío (3500-1400 años AP sensu Iriondo y Kröhling, 1995) que culmina la secuencia sedimentaria del área.

Resultados sedimentológicos indican que el loess fue originado a partir de una suspensión eólica, la que representa el 70 a $90 \%$ de los mecanismos de transporte identificados (Kröhling y Orfeo, 2002). La mezcla de materiales volcaniclásticos alterados y frescos, en la fracción arena y limo de la Formación Tezanos Pinto, evidencia la existencia de fuentes de procedencia múltiples: un aporte directo de lluvia de cenizas y uno indirecto por removilización de depósitos volcaniclásticos andinos. Una fuente secundaria está integrada por minerales procedentes de las altas cuencas de los ríos Paraná y Uruguay (Kröhling et al., 2006).

\section{MATERIALES Y MÉTODOS}

En el presente trabajo se seleccionó el sector sudoccidental del área de distribución de la Formación Tezanos Pintos en la provincia de Entre Ríos (Fig. 1), la cual se encuentra limitada por la margen izquierda del valle del Río Paraná, en donde se relevaron de NO a SE los siguientes perfiles, que corresponden a las mejores exposiciones de loess primario $=$ Diamante Norte $(\mathrm{PDN})$, Las Cuevas (PLC), Gauchito Gil (PGG) y Cantera Vialmat (PVM).

Las muestras se obtuvieron sobre los perfiles limpios, con muestreos equidistantes cada $10 \mathrm{~cm}$ promedio entre muestras, totalizando en su conjunto 44 muestras (Fig. 1), de aproximadamente $250 \mathrm{gr}$, que fueron incorporadas a la colección de muestras sedimentarias del Laboratorio de Paleobotánica del CICYTTP-Diamante. Para el proce- samiento se siguieron las pautas básicas para concentrar microrrestos silíceos de acuerdo a la metodología que propuso Zucol et al. (2010b), la cual de manera resumida puede describirse como el secado, molienda y tamizado grueso del material proveniente del campo, eliminación de sales solubles (con agua destilada en frío y luego en caliente), de carbonatos, cementos y barnices (con ácido clorhídrico en frío y en caliente) y de materia orgánica (agregando agua oxigenada 100 volúmenes al 30\%); dispersión de las arcillas (utilizando hexametafosfato de sodio); separación granométrica para la obtención de tres fracciones (fina -diámetro entre 5 y $53 \mu \mathrm{m}$-, media -diámetro entre 53 y $250 \mu \mathrm{m}-$ y gruesa -diámetro mayor a $250 \mu \mathrm{m}-$ ), separación densimétrica (con politungstato de sodio como líquido pesado) y por último su montado en preparados fijos (Bálsamo de Canadá) y líquidos (aceite de inmersión).

La muestra mínima resultó de 400 fitolitos/muestra, obtenida mediante recuentos progresivos de variabilidad de 5 muestras tomadas al azar, mientras que para los recuentos se utilizó un conjunto de morfotipos fitolíticos compendiados en una clasificación ad-hoc cuya denominación, acrónimo, como así también, sus equivalencias con otras clasificaciones y descriptores se detallan en la Tabla 1 (Erra, 2010b, 2011).Estas últimas publicaciones se utilizaron asimismo para establecer las afinidades botánicas de los morfotipos fitolíticos. Las observaciones microscópicas fueron realizadas en un microscopio Hokenn modelo WPB 100 y las fotografías fueron obtenidas con una cámara digital Sony DSC-W30.

Para el procesamiento numérico y graficación de la información se utilizó el programa POLPAL, Numerical Analysis (Walanus y Nalepka, 1999), lo cual se ha resumido mediante la confección de diagramas fitolíticos (distribución vertical de la abundancia relativa de cada morfotipo a lo largo del perfil), el dendrograma de vinculación de estas asociaciones fitolíticas, mediante la aplicación del método CONISS -Constrained incremental sum of squares cluster analysis (Grimm, 1991) - que permitió establecer la zonificación de cada perfil y el aporte de variabilidad de cada morfotipo de acuerdo al análisis de variabilidad que realiza este programa (Walanus y Nalepka, 1999). Con la finalidad de comparar las asociaciones de cada perfil se realizó el análisis de correspondencia (CA) de las asociaciones fitolíticas de las muestras, mediante el programa PAST (Hammer et al., 2007). En ambos casos, las matrices básicas de datos (MBD) confeccionadas utilizaron como OTUS a las muestras (asociaciones fitolíticas) obtenidas en los perfiles 


\begin{tabular}{|c|c|c|c|c|}
\hline Morfotipos & Acrónimos & (g) & (f) & Equivalencias y afinidad botánica \\
\hline \multirow[t]{9}{*}{ Aguzados } & Acac & Ac01 & Ps01 & \multirow{9}{*}{$\begin{array}{l}\text { Aculeolita (a), Point-shaped class ( } b \text { y } d \text { ), Point-shaped (e y f), } \\
\text { Aguzados (g) } \\
\text { Poaceae p.p. (a) } \\
\text { No diagnóstico Nd01 (Figs. 3 y 5) }\end{array}$} \\
\hline & Acci & Ac02 & Ps02 & \\
\hline & Accu & Ac04 & Ps04 & \\
\hline & Acfi & Ac05 & Ps05 & \\
\hline & Acmd & Ac06 & Ps06 & \\
\hline & Acpi & Ac08 & Ps08 & \\
\hline & Acnt & Ac07 & Ps07 & \\
\hline & Acro & Ac09 & Ps09 & \\
\hline & Acuc & Ac10 & Ps10 & \\
\hline Radiados & Ass1 & As01 & Ss01 & Cyperoides Cy p.p. (Figs. 3 y 5) \\
\hline Bacilares & Baca & $\mathrm{Ba01}$ & -- & No diagnóstico Otros p.p.(Figs. 3 y 5 ) \\
\hline \multirow[t]{2}{*}{ Prismáticos cortos } & Bron & Bro1 & Be01 & \multirow{2}{*}{$\begin{array}{l}\text { Braqueolita (a), Brief Elongate (e y f), Brevilitas (g). No diagnóstico } \\
\text { Nd02 p.p. (Figs. } 3 \text { y } 5 \text { ) }\end{array}$} \\
\hline & Brps & $\mathrm{BrO2}$ & $\mathrm{Be} 02$ & \\
\hline En forma de cruz & Chbr & Ch01 & $\mathrm{CrO1}$ & $\begin{array}{l}\text { Euhalteriolita cruciformata (a), Panicoids class p.p. (b y d), Cross } \\
\text { shaped (e), Cross ( } f \text { ), Cruces (g). Poaceae: Panicoideae (b) Pa01 (Figs. } \\
3 \text { y 5) }\end{array}$ \\
\hline \multirow[t]{3}{*}{ En forma de silla de montar } & Doae & Sm01 & Sa01 & \multirow{3}{*}{$\begin{array}{l}\text { Doliolita (a), Chloridoids class (b y d), Saddle (b, c, e, d y f), Sillas de } \\
\text { montar (g). Poaceae: Chloridoideae (b) Ch (Figs. } 3 \text { y 5) }\end{array}$} \\
\hline & Doel & $\mathrm{Sm} 02$ & $\mathrm{SaO2}$ & \\
\hline & Doob & $\mathrm{Sm03}$ & $\mathrm{SaO3}$ & \\
\hline \multirow{3}{*}{$\begin{array}{l}\text { Originados en elementos de } \\
\text { conducción }\end{array}$} & Dpan & Du01 & $\mathrm{Ce} 01$ & \multirow{3}{*}{$\begin{array}{l}\text { Cylindrical conduction element (e yf), Elementos de conducción (g). } \\
\text { No diagnóstico Nd08 (Figs. } 3 \text { y 5) }\end{array}$} \\
\hline & Dpla & Du02 & $\mathrm{CeO2}$ & \\
\hline & Dpsp & Du04 & $\mathrm{CeO4}$ & \\
\hline \multirow[t]{10}{*}{ Halteriformes bilobados } & Ehbi & $\mathrm{HaO1}$ & Dmo1 & \multirow{10}{*}{$\begin{array}{l}\text { Euhalteriolita (a), Panicoids class (b y d), Simply lobate and Panicoid } \\
\text { tipe (c), Bilobate (e), Dumbbell (f), Bilobados (g). Poaceae: Panicoi- } \\
\text { deae (b) Pa02 (Figs. } 3 \text { y 5) }\end{array}$} \\
\hline & Ehbo & $\mathrm{HaO2}$ & $\mathrm{Dm} 02$ & \\
\hline & Ehel & $\mathrm{HaO3}$ & -- & \\
\hline & Ehpc & $\mathrm{Ha05}$ & Dm05 & \\
\hline & Ehph & HaO6 & Dmo6 & \\
\hline & Ehtc & HaO7 & Dmo7 & \\
\hline & Ehtd & HaO8 & Dm08 & \\
\hline & Ehth & HaO9 & Dmo9 & \\
\hline & Ehtl & Ha10 & $D m 10$ & \\
\hline & Ehtp & Ha11 & $D m 11$ & \\
\hline \multirow[t]{7}{*}{ En forma de abanico } & Flbi & Fl01 & Fs01 & \multirow{7}{*}{$\begin{array}{l}\text { Flabelolita (a), Fan-shaped class ( } b \text { y d), Cuneiform bulliform cell } \\
\text { (e), Fanshaped (f), Flabelos (g). Poaceae (a y b) No diagnóstico Nd05 } \\
\text { (Figs. } 3 \text { y 5) }\end{array}$} \\
\hline & Flca & Fl02 & $\mathrm{FsO2}$ & \\
\hline & Flco & Fl03 & Fs03 & \\
\hline & Flel & Fl04 & Fs04 & \\
\hline & Fleu & Fl05 & Fs05 & \\
\hline & Flex & Fl06 & & \\
\hline & Flhe & Fl07 & Fs07 & \\
\hline \multirow[t]{3}{*}{ Globulares } & $\mathrm{GlCl}$ & Gl01 & & \multirow{3}{*}{$\begin{array}{l}\text { Globulolita (a), Spherical class (d), Globular y Macroglobular (e y f), } \\
\text { Globulares y Macroglobulares (g). Palmaceae p.p. (a) Dicotiledòneas } \\
\text { p.p. (d), Ar (Figs. 3 y 5) }\end{array}$} \\
\hline & Glee & Gl02 & Gl02 & \\
\hline & Glep & Gl03 & Gl03 & \\
\hline
\end{tabular}




\begin{tabular}{|c|c|c|c|c|}
\hline \multirow[t]{4}{*}{ Morfotipos } & Acrónimos & (g) & (f) & \multirow[t]{4}{*}{ Equivalencias y afinidad botánica } \\
\hline & Glse & Gl04 & G104 & \\
\hline & Glsp & Gl05 & G105 & \\
\hline & Mgsp & Mg01 & Mg01 & \\
\hline \multirow[t]{4}{*}{ Irregulares prismáticos } & Locl & $\operatorname{Ln} 01$ & Fu01 & \multirow{4}{*}{$\begin{array}{l}\text { Longolita (a), Fusiform (e y f), Fusiformes (g). Podostomaceae (a), } \\
\text { Pod (Figs. } 3 \text { y 5) }\end{array}$} \\
\hline & Loma & LnO2 & Fu02 & \\
\hline & Lona & $\operatorname{Ln} 03$ & Fu03 & \\
\hline & Love & Ln04 & Fu04 & \\
\hline Lobulados & Los1 & $L b 01$ & $L b 01$ & Lobular (f), Lobulados (g). Dicotiledòneas (d). \\
\hline \multirow[t]{2}{*}{ Multicavados } & $\mathrm{Mccl}$ & Mc01 & Mc01 & \multirow[t]{2}{*}{ No diagnóstico Nd07 (Figs. 3 y 5) } \\
\hline & Mcrc & Mc03 & Mc03 & \\
\hline \multirow[t]{11}{*}{ Prismáticos elongados } & Mips & Mi01 & Me01 & \multirow{11}{*}{$\begin{array}{l}\text { Euprismatolita (a), Elongate class (b y d), Micro-elongate y Elongate } \\
\text { (e y f), Prismáticos elongados cortos y largos ( } g \text { ) No diagnóstico } \\
\text { Nd03, excepto Mips No diagnóstico Nd02 p.p. (Figs. } 3 \text { y 5) }\end{array}$} \\
\hline & Mpan & Mp01 & El01 & \\
\hline & Mpdf & Mp02 & EIO2 & \\
\hline & $M p d t$ & Mp03 & EIO3 & \\
\hline & Mpel & Mp04 & ElO4 & \\
\hline & Mpex & Mp05 & EI05 & \\
\hline & Mpis & Mp06 & El06 & \\
\hline & Mpmd & Mp07 & El07 & \\
\hline & Mpon & Mp08 & EI08 & \\
\hline & Mpps & Mp09 & El09 & \\
\hline & Mpsr & Mp10 & El10 & \\
\hline \multirow{3}{*}{$\begin{array}{l}\text { Prismáticos elongado } \\
\text { ramificados }\end{array}$} & Npse & Np01 & $E b 01$ & \multirow[t]{3}{*}{ No diagnóstico Nd04 (Figs. 3 y 5) } \\
\hline & Npma & $\mathrm{Np02}$ & $E b 02$ & \\
\hline & Npre & $\mathrm{Np03}$ & $E b 03$ & \\
\hline Halterios estipoideos & Phcr & Mh01 & -- & $\begin{array}{l}\text { Stipa type dumbbell (cye), Halterio tipo stipa (g). Poaceae: Pooideae; } \\
\text { Stipeae (c) St (Figs. } 3 \text { y 5) }\end{array}$ \\
\hline \multirow[t]{4}{*}{ Halterios complejos } & Phct & Ph01 & PI01 & \multirow{4}{*}{$\begin{array}{l}\text { Plurihalteriolita (a), Polylobate (e y f), Polilobados (g). Poaceae: } \\
\text { Panicoideae (a y b) Pa03 (Figs. } 3 \text { y 5) }\end{array}$} \\
\hline & Phin & $\mathrm{Ph02}$ & PIO2 & \\
\hline & Phtr & Ph03 & PI03 & \\
\hline & Phve & Ph04 & -- & \\
\hline \multirow[t]{5}{*}{ Cónicos } & Piap & Pi01 & Co01 & \multirow{5}{*}{$\begin{array}{l}\text { Pileolita (a), Conical (f), Cónicos (g). Cyperaceae (a y h) Cy p.p. (Figs. } \\
3 \text { y 5) }\end{array}$} \\
\hline & Pipc & Pi02 & $\mathrm{CoO2}$ & \\
\hline & Pior & Pi03 & -- & \\
\hline & Pipa & Pi04 & -- & \\
\hline & Pipe & Pi05 & -- & \\
\hline \multirow[t]{8}{*}{ En forma de conos truncados } & Stae & Ct01 & Ct01 & \multirow{8}{*}{$\begin{array}{l}\text { Estrobilolita (a), Chionocloid class and truncated cones (d), } \\
\text { Truncated cones (f), Conos truncados (g). Poaceae: Arundinoideae } \\
\text { (d) Da (Figs. } 3 \text { y 5) }\end{array}$} \\
\hline & Stbi & $\mathrm{Ct02}$ & $\mathrm{CtO2}$ & \\
\hline & Stcm & $\mathrm{Ct03}$ & $\mathrm{CtO3}$ & \\
\hline & Stct & Ct04 & Ct04 & \\
\hline & Stel & Ct05 & Ct05 & \\
\hline & Stex & Ct06 & Ct06 & \\
\hline & Stgr & Ct07 & Ct07 & \\
\hline & Stax & Ct08 & Ct08 & \\
\hline
\end{tabular}




\begin{tabular}{|c|c|c|c|c|}
\hline \multirow[t]{2}{*}{ Morfotipos } & Acrónimos & (g) & (f) & \multirow[t]{2}{*}{ Equivalencias y afinidad botánica } \\
\hline & Sttu & $C+10$ & $C+10$ & \\
\hline \multirow[t]{4}{*}{ Poliédricos } & Sxs1 & Sx01 & Po01 & \multirow{4}{*}{$\begin{array}{l}\text { Polyhedral epidermal class (d), Parallepipedal bulliform cell (e), } \\
\text { Polyedrical (f), Poliédricos (g). No diagnóstico Nd06 (Figs. } 3 \text { y 5) }\end{array}$} \\
\hline & Sxs2 & $5 \times 02$ & Po03 & \\
\hline & Sxs3 & $5 \times 03$ & Po05 & \\
\hline & Sxs6 & $5 \times 06$ & Po06 & \\
\hline \multirow[t]{8}{*}{ Otros } & ZC1 & Sc01 & Ts01 & $\begin{array}{l}\text { Festucoid class and Festucoid boat (d), Sinuate trapezoid (f), En for- } \\
\text { ma de bote (g). Poaceae: Pooideae; Festuceae (b y d) Poo p.p. (Figs. } \\
3 \text { y 5) }\end{array}$ \\
\hline & $Z C 2$ & Ro01 & Rno1 & \multirow{2}{*}{$\begin{array}{l}\text { Pooid class (b), Festucoid class (d), Rondel (e y f), Redondeados (g). } \\
\text { Poaceae: Pooideae; Pooeae (by d) Poo p.p. (Figs. } 3 \text { y 5) }\end{array}$} \\
\hline & ZC3 & Ro02 & RnO2 & \\
\hline & ZC4 & Tr01 & Tr01 & $\begin{array}{l}\text { Triangular (e y f), Triangulares (g). No diagnóstico Otros p.p. (Figs. } \\
3 \text { y } 5 \text { ) }\end{array}$ \\
\hline & ZC5 & Sn05 & -- & No diagnóstico Otros p.p. (Figs. 3 y 5 ) \\
\hline & ZC6 & At02 & Ta01 & $\begin{array}{l}\text { Tracheid class (d), Short Tracheid (f). No diagnóstico Otros p.p. (Figs. } \\
3 \text { y 5) }\end{array}$ \\
\hline & ZC7 & Sn01 & Pg01 & $\begin{array}{l}\text { Poligonal plate (f). Cyperaceae y Commelinaceae (a, he i) No } \\
\text { diagnóstico Otros p.p. (Figs. } 3 \text { y } 5 \text { ) }\end{array}$ \\
\hline & ZC10 & $\mathrm{SnO3}$ & Pp01 & Prismatic papillose (f). No diagnóstico Otros p.p. (Figs. 3 y 5) \\
\hline
\end{tabular}

(a) Bertoldi de Pomar (1971), (b) Twiss (1992), (c) Fredlund y Tieszen (1994), (d) Kondo et al. (1994), (e) ICPN (2005), (f) Zucol et al. (2010 a y referencias en esta contribución), (g) Patterer et al. (2011), (h) Fernández Honaine et al. (2009) e (i) Eichhorn et al. (2010).

y como variables a las abundancias de morfotipos presentes en cada muestra (datos continuos discretos).

Las dataciones por OSL de sedimentos referidas en este trabajo fueron obtenidas por el Prof. Steven Forman ( $L u$ miniscence Dating Research Laboratory, University of Illinois at Chicago, USA; multiple aliquot regenerative dose technique -Jain et al., 2003).

\section{RESULTADOS}

\section{Descripción estratigráfica de los perfiles estudiados}

Diamante Norte (Fig. 1.1. Diamante Norte). El perfil muestreado se ubica al norte de la localidad de Diamante. La facies eólica de la formación aflora con un perfil vertical de 3,60 m de potencia, sin base expuesta. Es un depósito masivo, friable, de color marrón. Está compuesto por limo con escasa arena muy fina y arcilla. El loess constituye el material parental del suelo actual, de 1,30 m de espesor.

Las Cuevas (Fig. 1.3 Las Cuevas). El perfil aflora sobre la margen sur del camino a la localidad de Las Cuevas, a pocos cientos de metros antes de llegar a la misma. La Formación Tezanos Pinto se muestra como un depósito dominantemente limoso, con escasa arena y arcilla, de color marrón, masivo y friable. Presenta macroporos finos y muy finos. Contiene algunas concreciones nodulares de $\mathrm{CaCO}_{3}$ puras. El loess alcanza 0,90 $\mathrm{m}$ de potencia aflorante, con base cubierta. Constituye el material parental de un suelo débilmente desarrollado, correspondiente al suelo actual, representado por el Horizonte $\mathrm{Bt}(0,70 \mathrm{~m}$ de espesor) y el Horizonte BC (0,30 $\mathrm{m}$ de espesor).

Gauchito Gil (Fig. 1.2 Gauchito Gil). El perfil aflora en la ruta de circunvalación de la ciudad de Victoria, con un espesor de 2,20 $\mathrm{m}$ sin base expuesta. Corresponde a la facies eólica de la unidad, compuesta por limo con contribución subordinada de arena muy fina y escasa arcilla y de color marrón, con marcada tendencia granodecreciente. En los centímetros inferiores aparecen abundantes concreciones carbonáticas, suprayacentes a una lente de ceniza volcánica que alcanza 0,35 $\mathrm{m}$ de espesor. Esta unidad apoya en discordancia sobre la Formación Puerto Alvear (Pleistoceno; Iriondo y Kröhling, 2008). El suelo actual alcanza 0,50 m de potencia.

Cantera Vialmat (Fig. 1.4 Cantera VialMat). El perfil se encuentra en las proximidades de la Localidad Rincón de Nogoyá. La facies eólica de la unidad alcanza 0,75 m de espesor, en discordancia erosiva sobre la Formación Puerto Alvear. Constituye un perfil vertical, compuesto por limo 
con escasa arena muy fina y arcilla, friable y masivo. En su techo se ha desarrollado un suelo de $0,90 \mathrm{~m}$ de potencia.

\section{Caracterización fitolítica}

Los perfiles estudiados presentan una composición uniforme, teniendo en cuenta los morfotipos más abundantes, que en gran parte resultan coincidentes con los descriptos para el área central. Las asociaciones que se encuentran en estos perfiles pueden caracterizarse por la elevada abundancia de fitolitos de mayor tamańo como los elongados prismáticos (de contorno lisos -Mips y Mpps- y ondulados -Mpon-), en forma de abanico (Flco y Fleu) y poliédricos (Sxs1). Mientras que entre los fitolitos de menor tamaño la asociación presentó elementos globulares esféricos equinados (Glsp), en forma de conos truncados (Stae, Stcm, Stel y Sttu), en forma de silla de montar (Doae), halteriformes (Ehbi) y halteriformes aquillados del tipo stipa (Phcr). Los fitolitos articulados se han presentado en baja frecuencia y por lo general resultaron de naturaleza graminoide y de origen epidérmico, formados por un escaso número de células silicificadas (Fig. 2.9-12). Se han observado también otros tipos de microrestos silíceos, tales como espículas de espongiarios, estomatocistes de Chrysostomataceae (Fig. 2.15-16) y diatomeas (Fig. 2.13-14 y 17-18).

Diamante Norte (Fig. 3.1). El perfil Diamante Norte se caracteriza por una marcada abundancia de elementos prismáticos elongados, principalmente de contornos lisos y ondulados (Fig. 2.6 y 2.5 respectivamente), los cuales se encuentran acompañados, entre los fitolitos de mayor tamańo, por elementos en forma de abanico (Fig. 2.7) y aguzados (Fig. 2.4), presentando un incremento en el sector medio del perfil; mientras que entre los fitolitos de menor tamańo los de forma de conos truncados (Fig. 4.21-22) resultan los más abundantes en toda la sección, al igual que los globulares (Fig. 4.9-10). Los mismos se encuentran asociados con fitolitos en forma de silla de montar (Fig. 4.17-18), bilobados (Fig. 4.3-4) y halteriformes del tipo stipa, presentes en porcentajes menores.

$\mathrm{El}$ análisis de las abundancias de los distintos elementos (Fig. 3.1) permite definir estas tendencias mediante el establecimiento de cuatro zonas= PDNA $(1138-1143)$ con una marcada abundancia de fitolitos en forma de conos truncados, en forma de silla de montar y bilobados, una menor abundancia de fitolitos globulares equinados, prismáticos elongados, en forma de abanico y aguzados; PDNB (1144-1147) con menor abundancia relativa de elementos en forma de conos truncados y un incremento de fitolitos globulares equinados, prismáticos elongados, poliédricos (Fig. 2.2) y en forma de flabelos; PDNC (1148-1150) con un conjunto de muestras caracterizadas por un incremento abrupto de los fitolitos globulares equinados, como así también los conos truncados, en forma de silla de montar y bilobados tipo stipa y PDND (1151-1160) con una sección inferior en donde las asociaciones se encuentran dominadas por fitolitos prismáticos elongados asociados a elementos poliédricos y los globulares equinados, mientras que en menor medida se encuentran los de forma de conos truncados, bilobados, halteriformes tipo stipa y en forma de silla de montar y una sección superior en donde decrecen tanto los fitolitos elongados prismáticos como los elementos poliédricos y globulares equinados y se incrementan los fitolitos en forma de conos truncados, los bilobados, en forma de silla de montar, halteriformes tipo stipa, como así también, los aguzados.

A esta zonificación y de acuerdo a los componentes principales obtenidos (con un aporte de $\mathrm{Cp} 1=31 \%, \mathrm{Cp} 2=$ $16 \%, \mathrm{Cp} 3=9 \%$ de la variabilidad total) que se usaron para establecer estas secciones de perfil contribuyen distintos morfotipos. Para el caso del Cp1, que es el que demarca la diferenciación de estas cuatro zonas, contribuyen mayoritariamente las abundancias de los morfotipos en forma de silla de montar (Doae y Doob), bilobados (Ehbi, Phin y $\mathrm{Phtr}$ ), elongados prismáticos de contorno liso (Mpps), en forma de conos truncados (Stcm y Sttu), poliédrico (Sxs1) y fitolitos de formas triangulares (ZC4). Por su parte al $\mathrm{Cp} 2$, que permite diferenciar también estas zonas y parte de las variaciones internas de las PDNA, PDNB y PDND, contribuyen las abundancias de los morfotipos aguzados aciculares (Acci), bilobados (Ehbo), en forma de abanico (Flco, Fleu y Flex), elongados prismáticos de contorno liso y ondulado (Mpon y Mpps), en forma de conos truncados (Stae, Stcm y Stel) y fitolitos de formas triangulares (ZC4). Mientras que el Cp3 establece una separación entre las zonas inferiores, medias (PDNB y PDNC) y superior mediante el aporte de las abundancias de los morfotipos aguzados (Acac, Acci y Acfi), bilobados (Ehel, Ehtl y Phin), en forma de abanico (Fleu), halteriformes tipo stipa (Phcr), cónicos (Picr), en forma de conos truncados (Stae, Stct y Stel), poliédricos (Sxs2) y fitolitos de formas triangulares (ZC4).

Gauchito Gil (Fig. 5.1). En este perfil, del mismo modo que en el anterior, las asociaciones fitolíticas se encontraron dominadas por la presencia de fitolitos prismáticos elongados principalmente de contornos lisos y en menor abun- 
dancia ondulados (Fig. 2.6 y 5 respectivamente), elementos en forma de conos truncados y globulares (Fig. 4.12-13), mientras que, en menor frecuencia si bien presentes en toda la secuencia, se hallaron también fitolitos en forma de silla de montar, halteriformes bilobados (Fig. 4.1-2) y estipoides. De la comparación de las asociaciones fitolíticas en detalle (Fig. 5.1) puede evidenciarse que tanto la muestra superior como la inferior poseen una composición diferencial de las restantes, y que en muchos casos comparten la presencia de determinados fitolitos en forma exclusiva (por ejemplo Ehpc, Ehtp, Flel, Los1, Phct y Stgr) mientras que otros resultan exclusivos de cada muestra. De acuerdo a sus abundancias resultó posible subdividir la secuencia en cuatro zonas= PGGA (1105) en donde además de los morfotipos ya referidos resultan característicos la presencia de Acuc, Baca, Ehel, Mpis, Npma, ZC2, ZC3 y ZC4, siendo notable la baja abundancia de elementos globulares espinosos (Glse) y una elevada abundancia de elementos poliédricos (Sxs1 y Sxs2); PGGB (1108-1106) con un marcado incremento de las formas bilobadas tanto las pa-
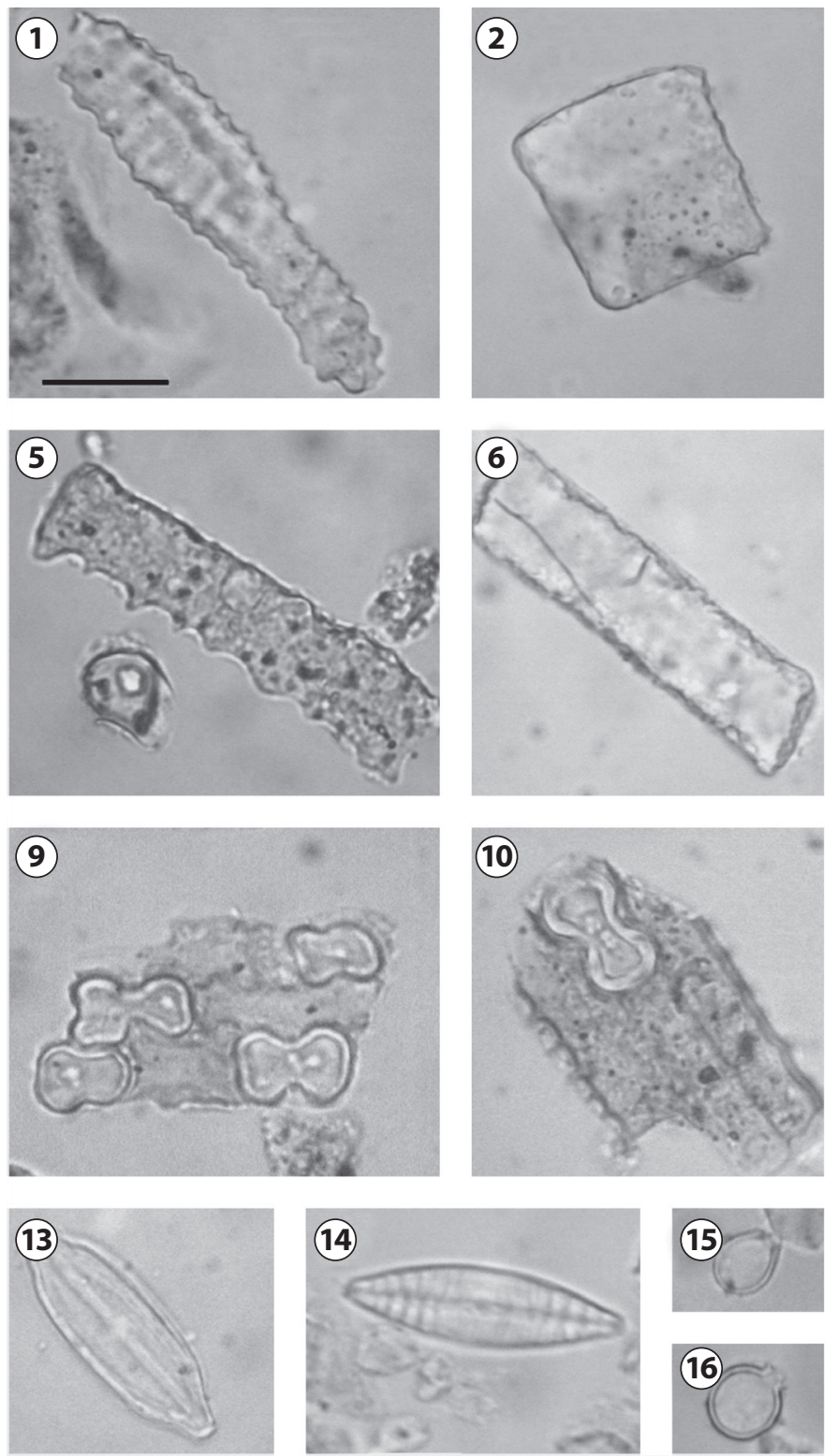
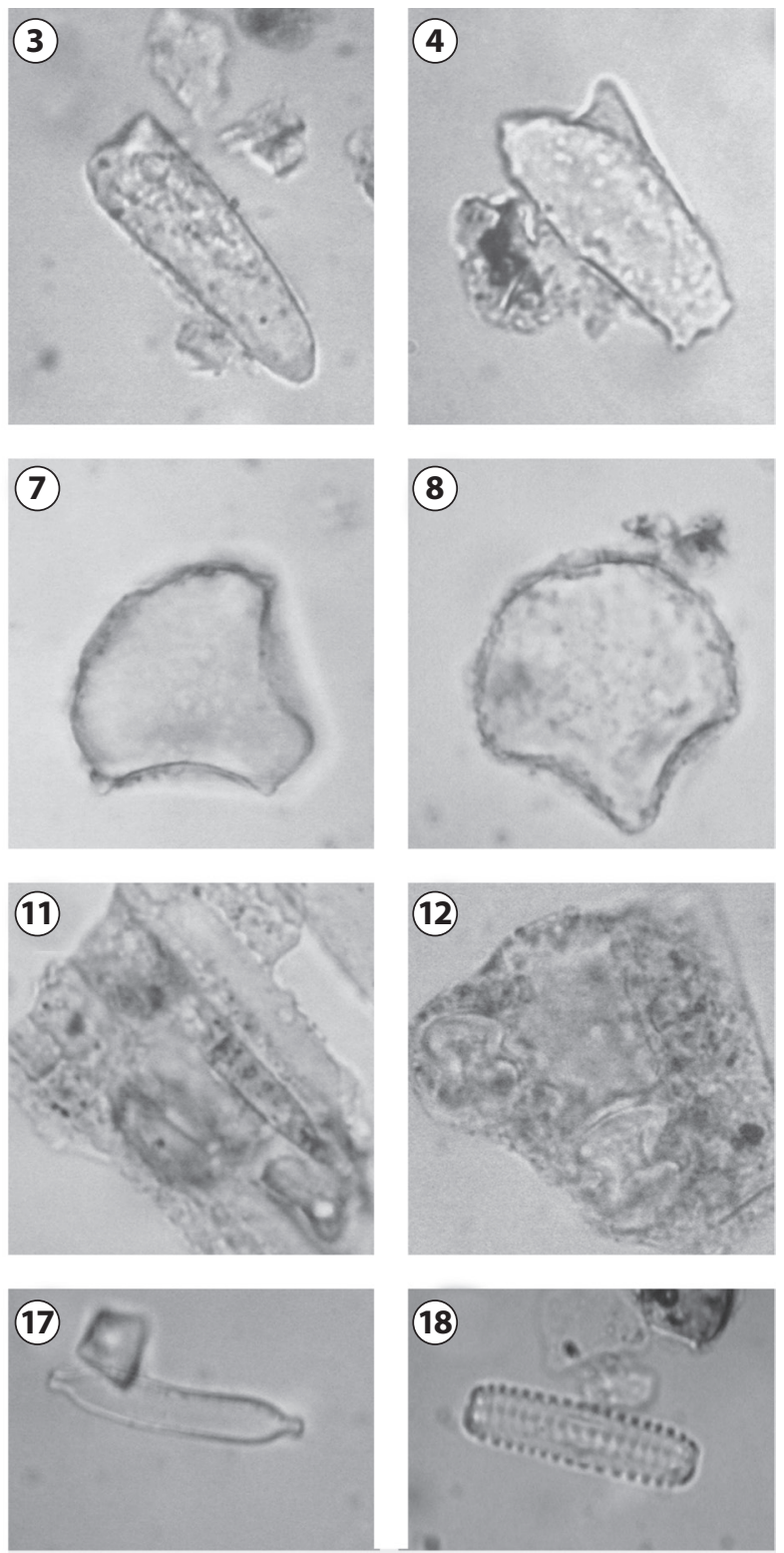

Figura 2. Microrestos silíceos hallados en la Formación Tezanos Pinto. Fitolitos no articulados. 1. Prismático irregular. 2. Poliédrico. 3-4. Formas aguzadas. 5-6. Fitolitos prismáticos. 7-8. En forma de abanico. 9-12. Fitolitos graminoides articulados de origen epidérmico. 13-14 y 17-18. Diatomeas. 15-16. Estomatocistes de chrysostomataceae. Escala gráfica $=20 \mu \mathrm{m}$. 


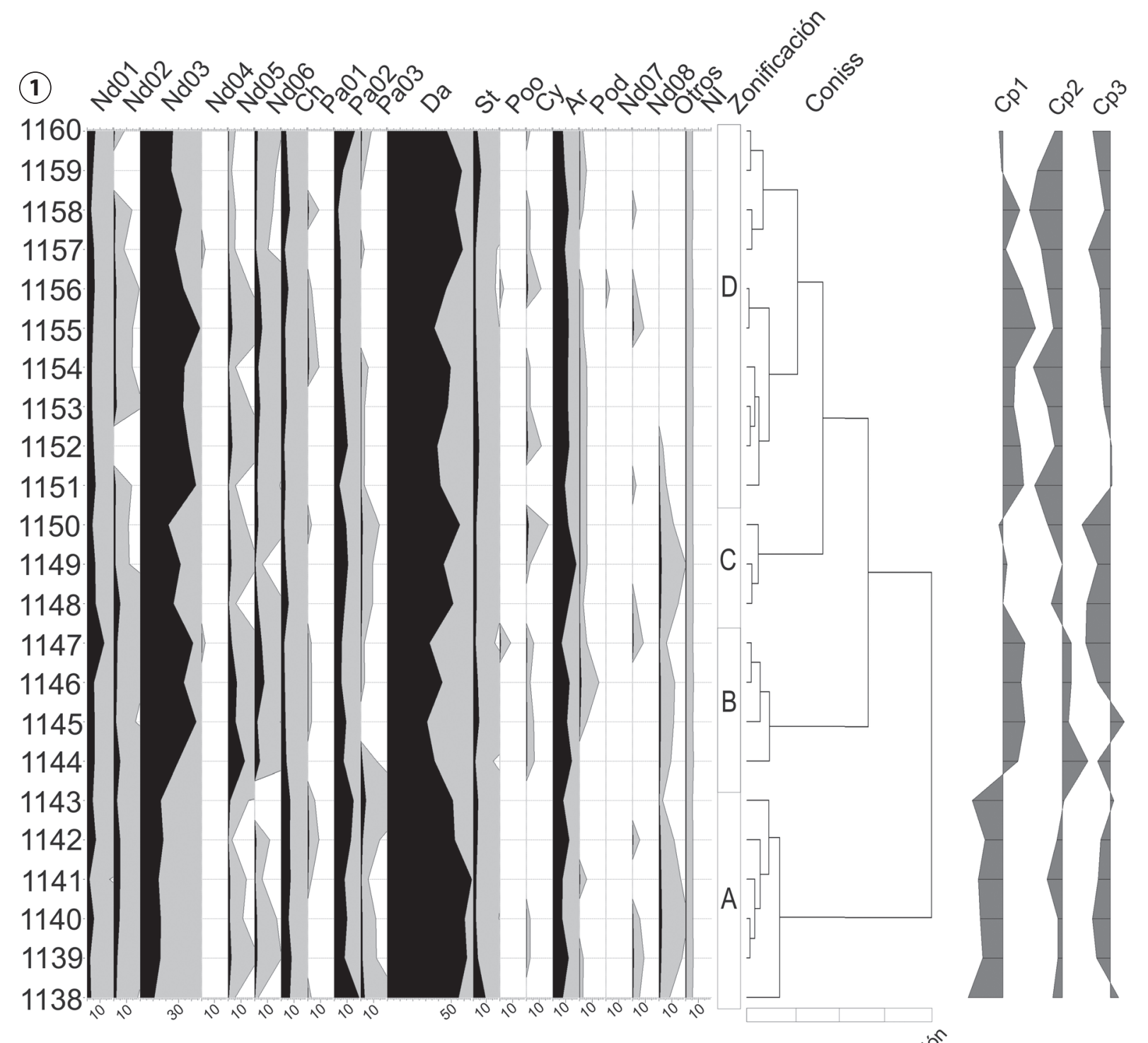

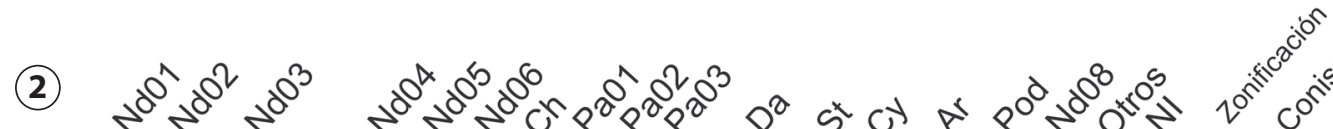
$e^{2} e^{2} e^{3}$ 1103
1102
1100
1099
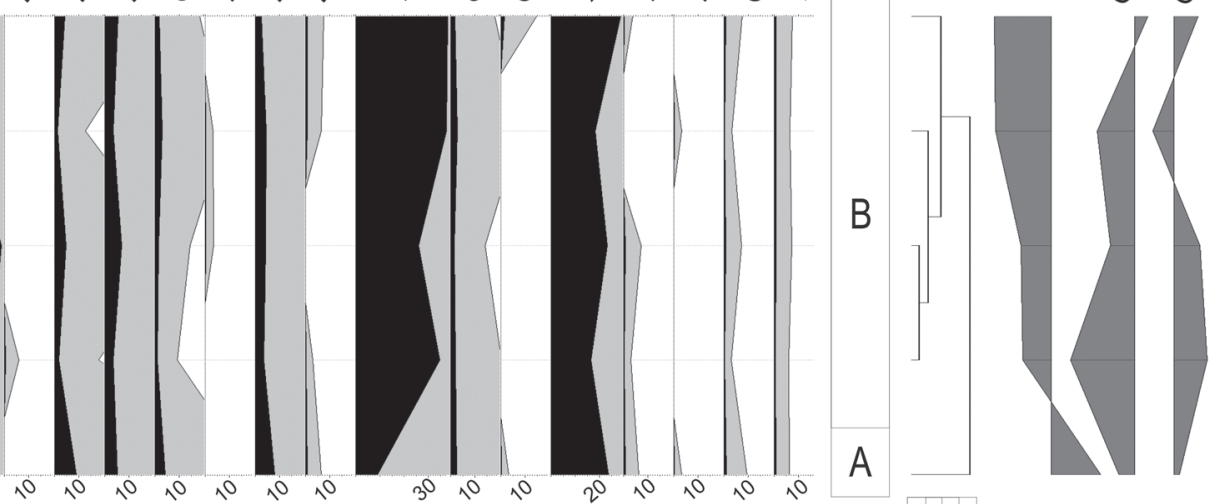

Figura 3. Diagrama fitolítico del perfil 1. Diamante Norte y 2. Vialmat, con la distribución de los principales morfotipos hallados (en recuentos). En negro = abundancia, en gris= exageración en factor 5. Dendrograma obtenido mediante Coniss análisis (utilizando el método de transformación Constrained single link with square root (SQRT)). Codificación de grupos de morfotipos por afinidad botánica de acuerdo a los especificado en Tabla 1 . 
nicoides como las estipoides y un gradual incremento de los fitolitos globulares espinosos hacia los niveles superiores de la zona, resultando muy abundantes también algunos tipos de conos truncados (Stcm y Stel), por otra parte resultaron escasos o ausentes los fitolitos poliédricos; PGGC (11091112) con un marcado incremento de los fitolitos globulares espinosos, la disminución de los fitolitos bilobados en correspondencia con el incremento de elementos en forma de silla de montar, en forma de conos truncados y la presencia de elementos poliédricos; y PGGD (1113), en donde se manifiesta un marcado incremento de los elementos bilobados y poliédricos en correspondencia con una menor abundancia de los fitolitos en forma de silla de montar y los globulares espinosos, mientras que por otra parte resultaron característicos de esta asociación los fitolitos Ass1, Flel, Glcl, Loma, Mpmd, y Sxs6. El establecimiento de esta zonificación se fundamenta en la obtención de los siguientes componentes principales (con un aporte de $\mathrm{Cp} 1=31 \%$, Cp2 $=23 \%, C p 3=18 \%$ de la variabilidad total): para el caso del Cp1, que es el que demarca la diferenciación de las zonas medias de la superior e inferior, contribuyen mayoritariamente las abundancias de los morfotipos bilobados elongados y múltiples (Ehel y Phtr), globulares esféricos lisos (Glsp), elongados prismáticos de contornos lisos y denticulados (Mpis y Mpdt), en forma de conos truncados equidimensionales (Stae), poliédricos (Sxs1 y Sxs2) y fitolitos cilíndricos cortos de sección circular (ZC2). Por otra parte es el Cp2 el que permite delimitar claramente cada una de las zonas anteriormente descriptas, mediante la variabilidad que mayoritariamente aportan los fitolitos aguzados (Acci y Acnt) (Fig. 2. 3), en forma de silla de montar (Doae), bilobados (Ehbi), en forma de abanico (Fleu), globulares esféricos (Glsp), irregulares (Lona) (Fig. 2 1), elongados prismáticos (Mpdt, Mpel, Mpis y Mpon), en forma de conos truncados equidimensionales (Stae), poliédricos (Sxs2) y fitolitos cilíndricos cortos de sección circular (ZC2). Por último, el Cp3 diferencia a la asociación fitolítica superior (PGGD) de las subyacente mediante el aporte de los fitolitos aguzados (Acci y Acro), bilobados elongados (Ehel), globulares esféricos (Glsp y Glse), elongados prismáticos (Mpdt y Mpon), en forma de conos truncados (Stae, Stcm y Sttu) y poliédricos (Sxs2).

Las Cuevas (Fig. 5.2). El perfil Las Cuevas se caracterizó por la elevada abundancia de ciertos morfotipos comunes en estos perfiles, como es el caso de los fitolitos globulares espinosos (Fig. 4.14-15), elongados prismáticos de contorno liso y ondulado y distintos tipos de elementos en forma de conos truncados (Fig. 4.23-25), si bien además resultaron muy frecuentes los fitolitos en forma de silla de montar (Fig. 4.16), distintos tipos de bilobados (Fig. 4.5-6), elementos aguzados y poliédricos. En lo que respecta a la distribución vertical de las asociaciones (Fig. 5.2) la mayor similitud entre las asociaciones se encuentra en la sección media, mientras que tanto en la sección inferior como en la superior se presentaron asociaciones fitolíticas unimuestrales de composición peculiar. Por lo cual de acuerdo al detalle de sus abundancias relativas se estableció la presencia de cuatro zonas= PLCA (1200), PLCB (1204-1201), PLCC (1205) y PLCD (1206), mediante el aporte de variabilidad diferencial que los distintos morfotipos realizaron a los componentes principales (con un aporte de $\mathrm{Cp} 1=$ $28 \%, \mathrm{Cp} 2=22 \%, \mathrm{Cp} 3=19 \%$ de la variabilidad total). La diferenciación de la PLCA (al igual que de la PLCD) se encuentra principalmente establecida por el Cp1, componente al cual aportan variabilidad los fitolitos en forma de silla de montar (Doae), bilobados (Ehbi y Ehtl), en forma de flabelo (Flco y Fleu), globulares (Glsp), irregulares (Lona), elongados prismáticos de contorno ondulado (Mpon) y en forma de conos truncados (Stae y Sttu). La PLCB se delimita a partir de los $\mathrm{Cp} 2$ (componente que también demarca la presencia de la PLCC) si bien más claramente por el Cp3, con una clara diferenciación interna de la asociación fitolítica de la muestra 1203 con respecto a las otras de esta zona. Al Cp2 los principales aportes lo realizan los elementos aguzados (Acac y Acro), bilobados (Ehel y Ehtd), en forma de flabelos (Flex), elongados prismáticos (Mpdt, Mpel, Mpon y Mpps) y en forma de conos truncados (Stcm, Stct y Stel). Mientras que al Cp3, por otra parte, contribuyen los fitolitos aguzados (Acac), en forma de silla de montar (Doae y Doel), bilobados elongados (Ehel), en forma de flabelos (Flco y Fleu), globulares (Glsp), bilobados tipos stipa (Phcr), en forma de conos truncados (Stex) y poliédricos (Sxs1).

Cantera Vialmat (Fig. 3.2). El perfil más distante y de menor potencia perteneciente a la Cantera Vialmat presentó una composición fitolítica más homogénea que los anteriores con una demarcada diferenciación de la asociación fitolítica de la muestra basal (1099) que se estableció como la zona PVMA, la cual posee una elevada riqueza de morfotipos en comparación con la sección suprayacente y una mayor abundancia relativa de muchos de los fitolitos presentes en gran parte del perfil como es el caso de los fitolitos aguzados, en forma de silla de montar (Fig. 4.19), bilobados, en forma de flabelos y elongados prismáticos, 
patrón que no ocurre con los fitolitos globulares, en forma de conos truncados (Fig. 4.20), bilobados tipo stipa y poliédricos. El análisis de las abundancias relativas que permitió diferenciar la zona inferior de la superior PVMB (1100-1103) se basó en el aporte diferencial de las abun- dancias fitolíticas a los componentes principales (con un aporte particular de $\mathrm{Cp} 1=53 \%$, $\mathrm{Cp} 2=23 \%$ y $\mathrm{Cp} 3=14 \%$ de la variabilidad total). Siendo en especial el Cp1 el que establece esta diferenciación de zonas, mediante el aporte de las formas aguzadas (Acci), bilobadas (Ehbo) (Fig. 4.7),
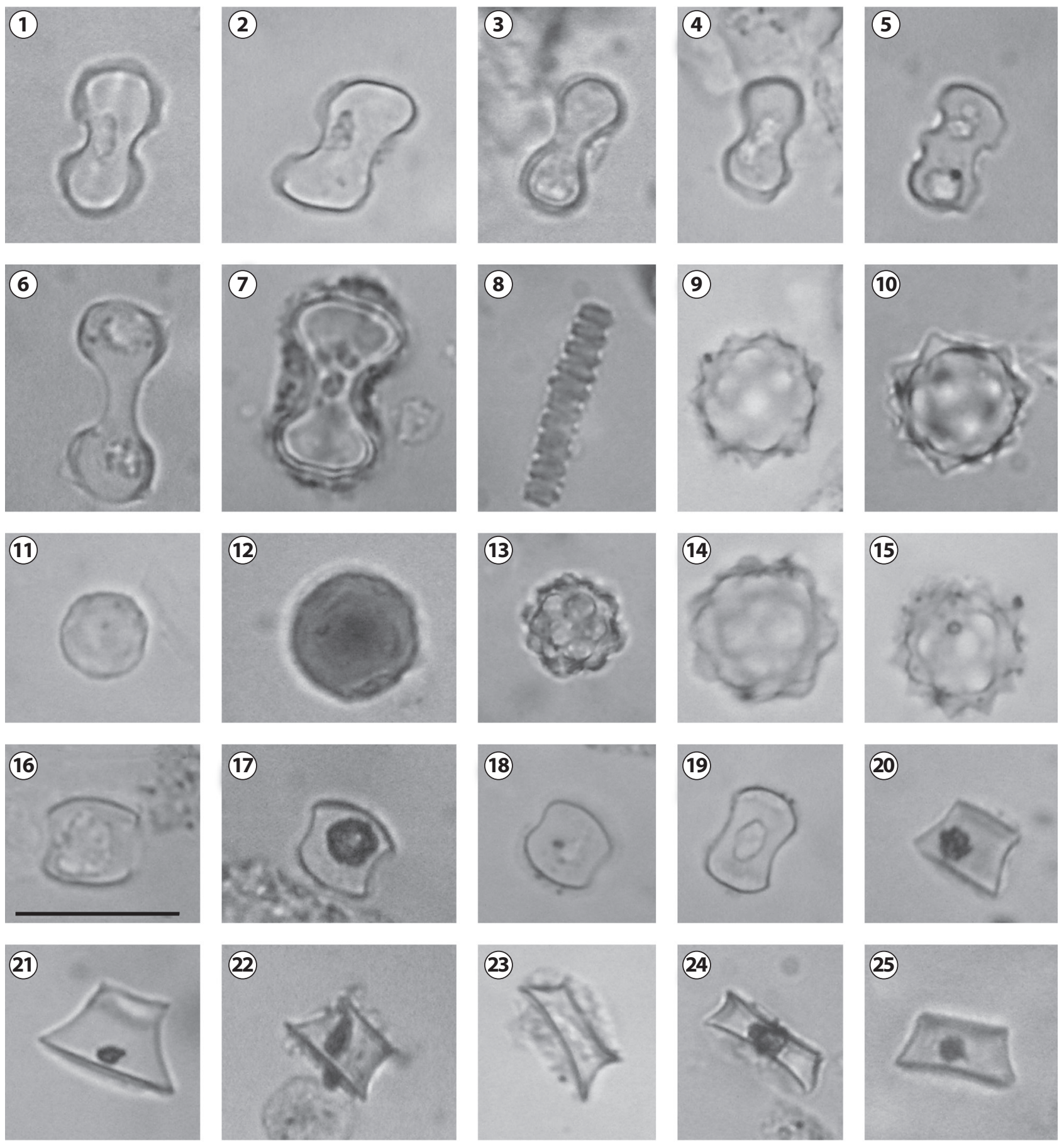

Figura 4. Fitolitos hallados en la Formación Tezanos Pinto. Fitolitos no articulados. 1-7. Fitolitos en forma de pesa de gimnasia. 8. Fitolito originado a partir de un elemento de conducción. 9-15. Distintos fitolitos globulares. 16-19. Fitolitos en forma de silla de montar. 20-25. Fitolitos en forma de cono truncados. Escala gráfica $=20 \mu \mathrm{m}$. 


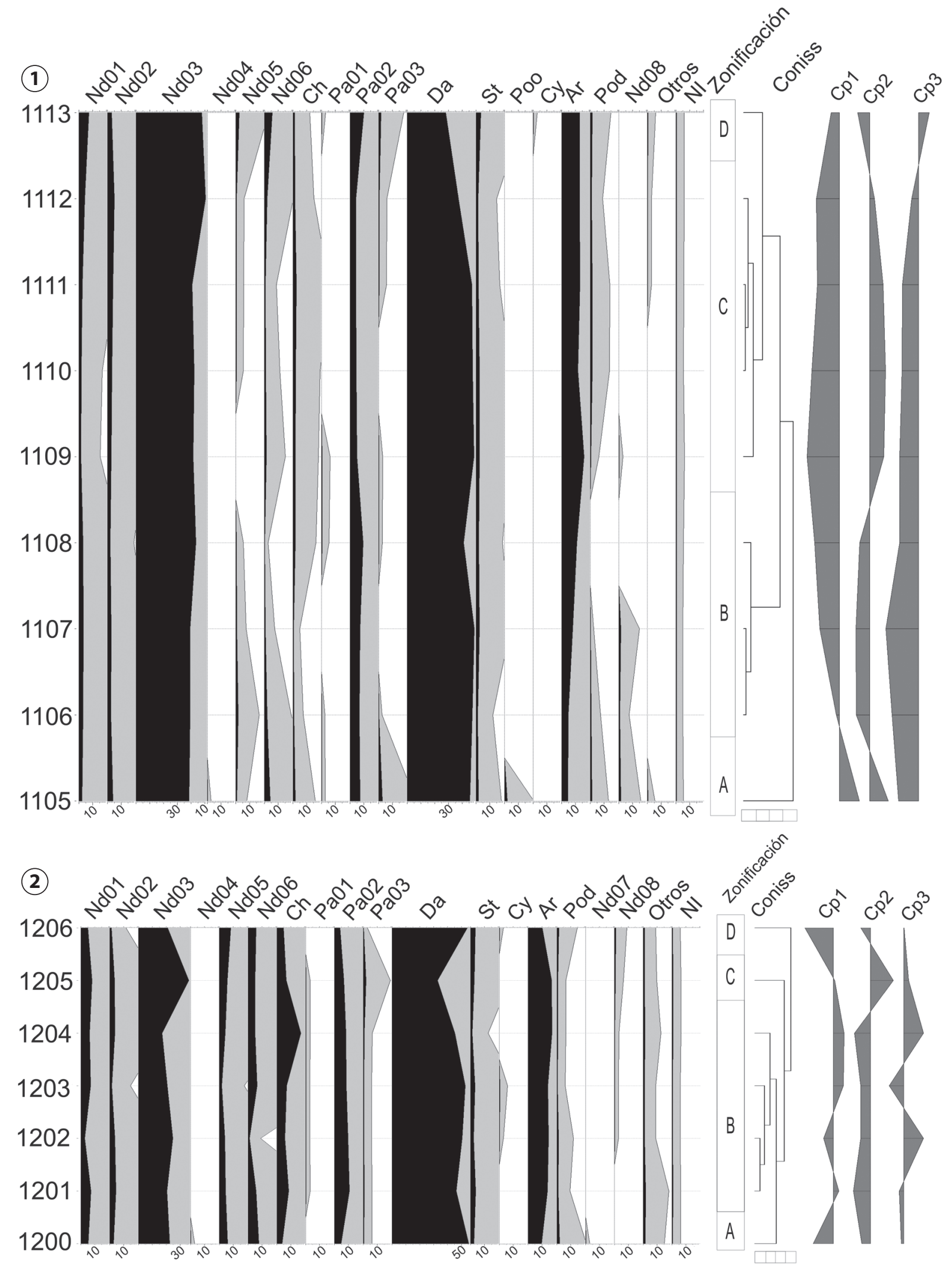

Figura 5. Diagrama fitolítico del perfil 1. Gauchito Gil y 2. Las Cuevas, con la distribución de los principales morfotipos hallados (en recuentos) En negro $=$ abundancia, en gris= exageración en factor 5 . Dendrograma obtenido mediante Coniss análisis (utilizando el método de transformación Constrained single link with square root (SQRT)). Codificación de grupos de morfotipos por afinidad botánica de acuerdo a los especificado en Tabla 1. 

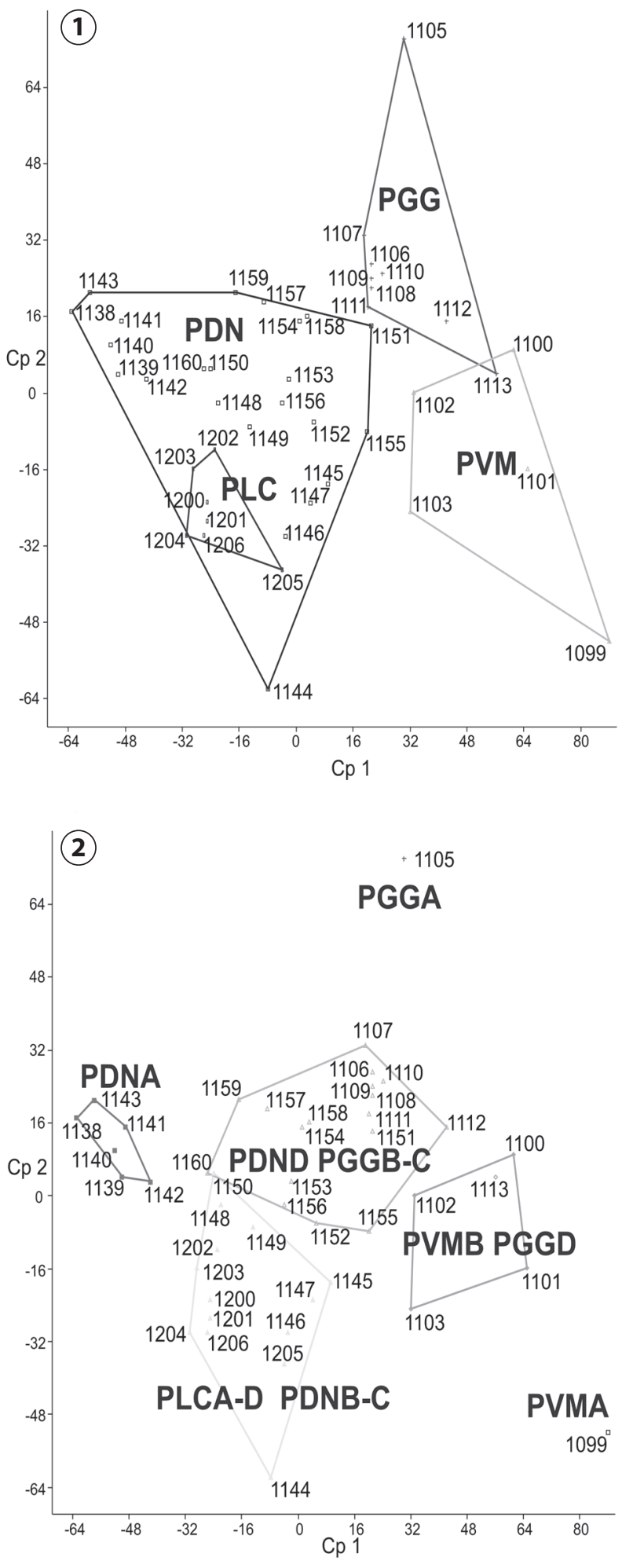

Figura 6. Análisis de correspondencia de las asociaciones fitolíticas de los cuatro perfiles analizados (codificación en el texto) considerando para su representación los ejes 1 y 2. 1. Agrupadas de acuerdo a cada perfil. 2. Agrupadas por zonas de cada perfil. en forma de flabelos (Flco y Fleu), globulares (Glse y Glsp (Fig. 4.11) y conos truncados (Stae, Stcm, Stel y Sttu). El Cp2 (conformado principalmente por el aporte de fitolitos aguzados (Acro), bilobados (EEhbi), en forma de flabelos (Flco y Fleu), globulares (Glse y Glsp), elongados prismáticos (Mpps), en forma de conos truncados (Stcm y Stel) y poliédricos (Sxs1 y Sxs2)) y el Cp3 (conformado mayoritariamente por el aporte de fitolitos aguzados (Acci), en forma de silla de montar (Doae), en forma de flabelos (Flco), elongados prismáticos (Mpps), bilobados tipo stipa (Phcr), en forma de conos truncados (Stae, Stcm y Stel) y poliédricos (Sxs1 y Sxs2) solo permiten diferenciar internamente la composición de las asociaciones fitolíticas de la PVMB.

\section{Análisis comparativo de las asociaciones fitoliticas}

El análisis de correspondencia de las asociaciones fitolíticas descriptas para estos cuatro perfiles (Fig. 6), considerando que en los mismos no existen diferencias en las características sedimentológicas al tratarse de la facies eólica típica, permitió estimar que las discrepancias internas halladas en las secciones de los cuatro perfiles corresponderían a variaciones locales que en algunos casos resultan vinculadas en diferentes perfiles. Teniendo en cuenta como conjunto a cada uno de los perfiles (Fig. 6.1), puede observarse una estrecha vinculación entre las asociaciones fitolíticas de los perfiles PDN y PLC, como así también a los perfiles PGG y PVM, lo cual se demarca por la variabilidad aportada por el Cp1 (10,66\% de la variabilidad), componente en el que participan en forma mayoritaria los siguientes morfotipos: Flel, Mpdf, Loma, Sxs6, Mpmd, Ass1, Accu, Ehph, Flbi, Ehpc, Doob, Flca y Npre. Mientras que por su parte el Cp2 $(5,98 \%$ de la variabilidad), diferencia mayoritariamente a las asociaciones fitolíticas de las muestras del perfil PGG de las del PVM, y en menor medida permite diferenciar los subgrupos internos de cada perfil; a este componente contribuye mayoritariamente la variabilidad de los siguientes morfotipos: ZC5, Pipa, Baca, Mpis, ZC2, Flca, Npma, Flhe, Stgr, ZC3, Acpi, Los1, Fleu, Ehpc, Dpan, Dpsp, Phct, Ehtp y Sxs3. Siendo algunos de estos elementos de importancia en ambos casos, como por ejemplo, Baca, Flca, Mpis, Ehpc, Pipa, Stgr y ZC2.

Esta misma distribución de las asociaciones fitolíticas puede analizarse teniendo en cuenta las distintas secciones obtenidas del análisis de cluster de cada perfil (Fig. 6.2). De este modo, las secciones basales de los perfiles PDN, PGG y PLC se separan claramente de las restantes asocia- 
ciones, tanto por el Cp1 (PDNA y PVMA) como por el Cp2 (PGGA); siendo este componente el que vincula a las asociaciones del perfil PLC (A-D) con la sección media del PDN (B-C), mientras que por otra parte presenta una estrecha relación entre las asociaciones superiores del PDN (D) con las secciones medias del PGG (B-C). Finalmente el Cp1 asocia la sección superficial de este perfil (PGGD) con las asociaciones de la sección medio superior del perfil PVM (B).

A nivel regional es clara la vinculación, desde el punto de vista composicional, de los perfiles PLC y PDN (p.p) con los perfiles La Juanita (PLJ), Protestante [PP (p.p.)] y Tezanos Pinto [PT (p.p.)] analizados previamente por Erra et al. (2011). Por otro lado, los perfiles PGG y PVM se asocian con el perfil Alvear (PA) que resulta atípico para el área noreste de su distribución. Estos últimos perfiles, se vinculan principalmente por las abundancias relativas de los morfotipos Ass1, Mpmd, Stgr, Sxs6, Npre, Mpis, Baca, Flex, Flca, Ehpc, ZC1, Flco y Acnt. Además, los elementos fitolíticos, como los de forma globular espinosos (Gl) o los fitolitos de forma cónica (Pi) los diferencia de los restantes perfiles (PGG, PA y PVM).

\section{AFINIDADES BOTÁNICAS}

El análisis fitolítico de las secuencias eólicas de la Formación Tezanos Pinto en el sector sudoeste de la provincia de Entre Ríos, permitió inferir que las asociaciones están dominadas por fitolitos graminoides y arecoides, los que se asociaron con menor frecuencia de elementos ciperoides, dicotiledóneos y podostemoides. Estos últimos, aunque se presentan con bajas abundancias, resultaron de importancia al momento de analizar interna y comparativamente los cuatro perfiles.

Los fitolitos ciperoides, representados principalmente por los morfotipos Ass1, Piap, Pipa, Pipe y ZC7, se presentan en la mayoría de los perfiles con excepción del PGG; mientras que por su parte los tipos podostemoides (Locl, Loma, Lona y Love) se hallaron en los cuatro perfiles. Los fitolitos lobulados de afinidad dicotiledónea (Los1) son escasos, presentándose principalmente en los niveles superiores de los perfiles con excepción del PVM.

Los fitolitos de palmeras (principalmente representados por los elementos globulares espinosos) son abundantes en todas las secciones analizadas, si bien su mayor abundancia relativa se presenta en el PVM.

En lo que respecta a los elementos graminoides, entre los de escaso poder diagnóstico se encuentran en forma abundante los fitolitos prismáticos, en forma de abanico $y$ poliédricos y, en menor frecuencia, pero con una gran variedad de formas, los elementos aguzados.

Entre los fitolitos graminoides de valor diagnóstico se encontraron distintos tipos de conos truncados de afinidad danthonioide, grupo mesotérmico de gramíneas que resultó presente en distintas variantes asociados a elementos microtérmicos como los estipoideos (fitolitos halteriformes estipoides aquillados) por una parte, como así también a elementos megatérmicos chloridoides (fitolitos en forma de silla de montar) y panicoides (fitolitos halteriformes bilobados, polilobados y en forma de cruz).

Para analizarse la distribución de estos grupos botánicos en forma comparativa en los perfiles estudiados, debe considerarse en primera medida que el PDN resultó ser el perfil de mayor potencia, quizás por ser el más cercano al área NO central que es donde se encuentra la localidad tipo, si bien cabe la posibilidad también que esto se deba a las características locales del paisaje. Si bien en los cuatro perfiles descriptos las muestras basales resultaron con una composición particular, en algunos casos diferenciadas de las asociaciones inmediatamente suprayacentes, en este perfil se presentó vinculada con la composición de la zona PDNA, la cual se diferencia de las restantes por poseer una marcada preponderancia de elementos chloridoides por sobre los panicoides entre sus elementos megatérmicos graminoides. Los elementos chloridoides decrecen en forma relativa en las zonas PDNB-C y se observa un incremento de los panicoides, como así también una mayor abundancia de los elementos ciperoides y podostemoides en la PDNC, sección esta que presenta una composición muy similar a la descripta para el PLC. Esta relación basal que demarca la presencia de elementos botánicos de condiciones xéricas ha sido también descripto para el perfil de la localidad tipo de la formación (PT, Erra et al., 2011).

$\mathrm{El}$ incremento de los elementos panicoides se hace más evidente en la zona PDND, en donde existe también un incremento de los elementos danthonioides debido a la mayor abundancia del tipo Stcm y también se evidencia una marcada abundancia de fitolitos ciperoides y podostemoides. Esta composición es compartida en gran parte con la del perfil PGG, en donde se observa también un predominio del tipo Stcm entre los elementos danthonioides y un paulatino incremento relativo de los morfotipos arecoides hacia las muestras superiores, siendo comparativamente abundantes en la muestra superior (1113), la cual presenta una composición vinculada a las observadas en el PVM. 
Las asociaciones fitolíticas de las muestras basales de los cuatro perfiles presentan composiciones particulares, que difieren entre sí, así como con las asociaciones inmediatamente superiores, lo cual hace pensar en una asociación transicional en la que las variaciones se hallan vinculadas a un carácter local del paisaje donde comenzaron a depositarse los sedimentos loéssicos. Mientras que la zona PDNA presenta una composición particular que no pudo observarse en los restantes perfiles del área sudoeste, aunque se encuentra en otros perfiles de mayor potencia y rangos temporales como el de la localidad tipo (PT).

\section{CONSIDERACIONES FINALES}

La presencia de abundantes elementos mesotérmicos y megatérmicos en todos los perfiles permite estimar la presencia de una vegetación de clima templado a templado cálido, conformando comunidades de los tipos de estepas a sabanas de palmeras durante la depositación de la Formación Tezanos Pinto (Pleistoceno tardío-Holoceno temprano). Un detalle pormenorizado posibilita establecer un sector basal con una proporción relativamente menor de palmeras y una marcada presencia de elementos áridos en el PDN, al igual que lo descripto para el PT (Erra $e t$ al., 2011). Condiciones que se hacen más húmedas en las secciones medias de la secuencia en donde se evidencia un incremento de elementos ciperoides y podostemoides; mientras que hacia las secciones superiores se presenta un mayor incremento de elementos panicoideos y arecoideos, los cuales conjuntamente con la presencia de abundantes fitolitos ciperoides y podostemoides se vinculan con una vegetación cálida desarrollada en ambientes con buena disponibilidad hídrica.

Si bien deben considerarse las variaciones climáticas actuales SO-NE, pueden compararse estos resultados con los descriptos para la región pampeana mediante distintos indicadores (Prado y Alberdi, 1999; Tonni et al., 1999, 2003; Quattrocchio et al., 2008; Tonello y Prieto, 2010) que concluyen que durante el Pleistoceno tardío en el sudeste pampeano se desarrollaron condiciones climáticas secas. Este lapso temporal corresponde al sector basal del perfil tipo (PT) y que se vincula con el sector basal del PDN en el área de estudio. Hacia la transición Pleistoceno tardíoHoloceno temprano, las condiciones climáticas varían de subhúmedas a húmedas y prevalecen hasta los 7500 años cal AP (Quattrocchio et al., 2008; Tonello y Prieto, 2010). Estas variaciones climáticas coinciden con las secuencias fitolíticas analizadas en el presente trabajo y están demarca- das por la abundancia de las gramíneas del tipo panicoideas como así también con el incremento de elementos arecoides. Estos registros son indicadores no solo de condiciones de humedad sino también de un clima templado cálido.

Tonello y Prieto (2010) describen para el Holoceno medio condiciones climáticas que varían según la región y que van desde condiciones húmedas (Quattrocchio et al., 2008) a subhúmedas secas con déficit hídrico (Prieto, 1996). En las secciones superiores de los perfiles estudiados PP, PLC y PLJ se observa un abrupto incremento de los morfotipos afines a las chloridoideas, los cuales comparten abundancias con los panicoides (elementos megatérmicos predominantes en las secciones inferiores) sugiriendo que estas variaciones de condiciones húmedas a subhúmedas secas también estuvieron presentes en esta región.

Las asociaciones fitolíticas descriptas en el loess de la Formación Tezanos Pinto en la provincia de Entre Ríos tienen un alto grado de homogeneidad. Análisis de detalle de perfiles de la unidad en el sector SO del área de distribución del loess permitieron hallar variaciones composicionales menores, que permiten vincularlas con la ubicación geomorfológica, considerando la cercanía de los perfiles aflorantes a la llanura aluvial del Río Paraná y a su complejo deltaico, lo cual ha permitido profundizar en el conocimiento de esta unidad.

La presencia de elementos afines a palmeras, ciperáceas y podostemáceas en asociación con fitolitos graminoides principalmente meso y megatérmicos, tipifican el ambiente de depósito del loess durante el UMG, en el borde noreste del Sistema Eólico Pampeano. Las principales variaciones halladas a lo largo de las secuencias analizadas responden a los patrones de variación ambiental descriptos para el sector más austral de la región pampeana.

\section{AGRADECIMIENTOS}

La presente contribución forma parte de la tarea de tésis doctoral que uno de los autores (GE) ha desarrollado en la Facultad de Ciencias Naturales y Museo de la Universidad Nacional de La Plata. Este trabajo se realizó mediante la financiación de los proyectos PICT 13864-2003 y 01762008 del Fondo para la Investigación Científica y Tecnológica (FonCyT) y Agencia Nacional de Promoción Científica y Tecnológica (ANPCyT).

\section{BIBLIOGRAFÍA}

Behrensmeyer, A.K. y Hook, R.W. 1992. Paleoenvironmental contexts and taphonomic modes in the terrestrial fossil record. En: A.K. Behrensmeyer, J.D.Damuth, W.A. DiMichele, R. Potts, H.-D. Sues y S.L. Wing (Eds.), Terrestrial Ecosystems Through Time. Universtiy of Chicago Press, Chicago, p. 15-136.

Bertoldi de Pomar, H. 1970. Fitolitos y Zoolitos. Su significado geológico en sedimentos continentales. Boletín de la Asociación Geológica de Córdoba 1: 21-31. 
Bertoldi de Pomar, H. 1971. Ensayo de clasificación morfológica de los silicofitolitos. Ameghiniana 8: 317-328.

Bertoldi de Pomar, H. 1980. Análisis comparativo de silicobiolitos de diversos sedimentos continentales argentinos. Revista Asociación Geológica Argentina 35: 547-557.

d'Orbigny, A. 1835. Synopsis terrestrium et fluviatilium molluscorum, in suoer American meridionalem itinere collectorum. Magazine Zoology 5, Classe 5 (61-62): 1-44.

d'Orbigny, A. 1842. Voyage dans l'Amérique Méridionale (le Brésil, la République orientale de l'Uruguay, le République Argentine, la Patagonie, le République du Chili, la République de Bolivia, la République du Perou), executé pendant les annés 1826, 1827, 1828, 1829, 1830, 1831, 1832 et 1833, volume 3, part 4. P. Bertrand, Paris, $561 \mathrm{p}$.

Eichhorn, B., Neumann, K. y Garnier, A. 2010. Seed phytoliths in West African Commelinaceae and their potential for palaeoecological studies. Palaeogeography, Palaeoclimatology, Palaeoecology 298: 300-310.

Erra, G. 2010a. [Estudio fitolítico de la Formación Tezanos Pinto (Pleistoceno Tardio-Holoceno Temprano) en la provincia de Entre Rios, Argentina. Tesis doctoral Facultad de Ciencias Naturales y Museo, Universidad Nacional de La Plata, La Plata, 277 p. Inédito].

Erra, G. 2010b. Asignación sistemática y paleocomunidades inferidas a partir del estudio fitolítico de sedimentos cuaternarios de Entre Ríos, Argentina. Boletín de la Sociedad Argentina de Botánica 45: 309-319.

Erra, G. 2011. Criterios metodológicos para el estudio y clasificación de fitolitos cuaternarios. Revista Historia Natural, Tercera serie 1: 45-62.

Erra, G., Zucol, A.F. y Kröhling D.M. 2011. Análisis fitolítico de la Formación Tezanos Pinto (loess del Pleistoceno Tardío- Holoceno Temprano) en el sector noroeste de su área de distribución en la provincia de Entre Ríos (Argentina). Revista Mexicana de Ciencias Geológicas 28: 298-312.

Fernández Honaine, M., Zucol, A.F. y Osterrieth, M.L. 2009. Phytolith analysis of Cyperaceae from the Pampean region, Argentina. Australian Journal of Botany 57: 512-523.

Frenguelli, J. 1930. Partículas de sílice organizadas en el loess y en limos pampeanos. Células silíceas de Gramíneas. Anales de la Sociedad Científica de Santa Fé 2: 64-109.

Fredlund, G.G., Tieszen, L.T., 1994, Modern phytoliths assemblages from North American Great Plains: Journal of Biogeography 21: 321-335

Grimm, E.C. 1991. TILIA Software. Illinois State Museum. Research and Collection Center, Springfield, IL, USA.

Gutiérrez, M.A., Martínez, G., Luchsinger, H., Grill, S., Zucol, A.F., Hassan, G.S., Barros, M.P., Kaufmann C.A. y Álvarez M.C. 2011. Paleoenvironments in the Paso Otero locality during Late PleistoceneHolocene (Pampean region, Argentina): an interdisciplinary approach. Quaternary International 245: 37-47.

Hammer, O., Harper, D.A.T. y Ryan, P.D. 2007. Past_Palaeontological Statistics, ver. 1.75, 86 pp. [versión original 2001, Palaeontologia Electronica 4(1): 1-9].

ICPN Work Group. 2005. International Code for Phytolith Nomenclature 1.0. Annals of Botany 96: 253-260.

Iriondo, M. 1980. El cuaternario de Entre Ríos. Revista de la Asociación de Ciencias Naturales del Litoral 11: 125-141.

Iriondo, M. 1998. Loess in Argentina: Temperate and Tropical. Excursions Guide No3. Province of Entre Ríos. International Union for Quaternary Reserch. International Joint Field Meeting, 12 p.

Iriondo, M. y Kröhling, D. 1995. El Sistema Eólico Pampeano. Subsecretaria de Cultura. Comunicaciones del Museo Provincial de Ciencias Naturales "Florentino Ameghino". Santa Fé 5: 1-68.

Iriondo, M. y Kröhling, D. 2007. Non-Classical Types of Loess. Sedimentary Geology 202: 352-368.

Iriondo, M. y Kröhling, D. 2008. Cambios ambientales en la cuenca del Uruguay (desde el Presente hasta dos millones de años atrás). Colección Ciencia y Técnica, Universidad Nacional del Litoral, Santa Fe, 360 p.

Jain, M., Botter-Jensen, L. y Singhvi, A.K. 2003. Dose evaluation using multiple-aliquot quartz OSL: test of methods and a new protocol for improved accuracy and precision: Radiation Measurements 37: 67-80.

Kondo, R., Childs, C. y Atkinson, I. 1994. Opal phytoliths of New Zealand. Maanaki Whenua Press, Lincoln, $85 \mathrm{p}$.

Kröhling, D. 1998. Excursion Guide N.2 North pampa (Carcarañá river basin, Santa Fe province). International Joint Field Meeting "Loess in Argentina: Temperate and Tropical" INQUA, loess Commission, 33 p.

Kröhling, D. 1999. Sedimentological maps of the typical loessic units in North Pampa, Argentina. Quaternary International 62: 49-55.

Kröhling, D. 2001. Quaternary paleosol - loessic sequences of southwestern Entre Ríos Province, Northern Pampa, Argentina. $G^{\text {th }}$ International Symposium and Field Workshop on Paleopedology in Mexico City (INQUA - ISSS). Abstracts: 27-28.

Kröhling, D. y Orfeo, O. 2002. Sedimentología de unidades loéssicas (Pleistoceno Tardío-Holoceno) del centro-sur de Santa Fe. Revista de la Asociación Argentina de Sedimentología 9: 135-154.

Kröhling, D., Passeggi, E., Zucol, A., Aguirre, M., Miquel, S. y Brea, M. 2006. Sedimentología y bioestratigrafía del loess pampeano del Pleistoceno tardío (Formación Tezanos Pinto) en el SO de Entre Ríos. $4^{\circ}$ Congreso Latinoamericano de Sedimentología y $11^{\circ}$ Reunión Argentina de Sedimentología (San Carlos de Bariloche), Resúmenes: 127.

Kröhling, D., Passeggi, E., Zucol, A., Erra, G., Aguirre, M., Miquel, S., González, A. y Brea, M. 2010. Multidisciplinary analysis of the last glacial loess at the NE of the Pampean aeolian system. $18^{\text {th }}$ International Sedimentological Congress (Mendoza), Abstract Volume: 518.

Osterrieth, M., Martínez, G., Gutiérrez, M. y Álvarez, M.F. 2008. Biomorfos de sílice en la secuencia pedoarqueológica del sitio Paso Otero 5, Buenos Aires, Argentina. En: A. Korstanje y P. Babot (Eds.), Matices Interdisciplinarios en Estudios Fitoliticos y de otros Microfósiles/ Interdisciplinary Nuances in Phytolith and other Microfossil Studies. BAR (British Archaeological Reports) International Series S1870, 9 Oxford, p. 77-90.

Patterer, N.I., Passeggi, E. y Zucol, A.F. 2011. Análisis fitolíticos de suelos del sudoeste de la Provincia de Entre Ríos (Argentina) como una herramienta para comprender sus procesos pedológicos. Revista Mexicana de Ciencias Geológicas 28: 132-146.

Prado, J.L. y Alberdi, M.T. 1999. The mammalian record and climatic change over the last 30,000 years in the Pampean Region, Argentina. Quaternary International 57/58: 165-174.

Prieto, A.R. 1996. Late Quaternary vegetational and climatic changes in the Pampa grassland of Argentina. Quaternary Research 45: 73-88.

Quattrocchio, M.E., Borromei, A.M., Deschamps,C.M., Grill, S.C. y Zavala, C.A. 2008. Landscape evolution and climate changes in the Late Pleistocene-Holocene, southern Pampa (Argentina): Evidence from palynology, mammals and sedimentology. Quaternary International 181: 123-138.

Teruggi, M. 1955. Algunas observaciones microscópicas sobre vidrio volcánico y ópalo organógeno en sedimentos pampeanos. Notas del Museo, Facultad de Ciencias Naturales y Museo, Universidad Nacional de Eva Perón, 18 Serie Geología 66: 17-26.

Teruggi, M.E. 1957. The nature and origin of Argentine loess. Journal of Sedimentary Petrology 27: 322-332.

Tonello M.S. y Prieto, A.R. 2010. Tendencias climáticas para los pastizales pampeanos durante el Pleistoceno tardío-Holoceno: estimaciones cuantitativas basadas en secuencias polínicas fósiles. Ameghiniana 47 (4): 501-514.

Tonni, E.P., Cione, A.L. y Figini, A.J. 1999. Predominance of arid climates indicated by mammals in the pampas of Argentina during the Late Pleistocene and Holocene. Palaeogeography, Palaeoclimatology, Palaeoecology 147: 257-281.

Tonni, E.P., Huarte, R.A., Carbonari, J.E. y Figini, A.J. 2003. New radiocarbon chronology for the Guerrero Member of the Luján Formation (Buenos Aires, Argentina): palaeoclimatic significance. Quaternary International 109-110: 45-48. 
Twiss, P.C. 1992. Predicted world distribution of $\mathrm{C}_{3}$ and $\mathrm{C}_{4}$ grass phytoliths. En: G. Rapp. Jr. y S.C. Mulholland (Eds.), Phytoliths Systematics. Emerging Issues. Advances in Archaelogical and Museum Science. Plenum Press, Nueva York, p.113-128.

Walanus, A. y Nalepka, D. 1999. POLPAL. Program for counting pollen grains, diagrams plotting and numerical analysis. Acta Palaeobotanica Suppl. 2: 659-661.

Zucol, A.F., Brea, M. y Passeggi, E. 2008. Los Estudios Fitolíticos en América del Sur, una Visión Retrospectiva. En: A. Korstanje y P. Babot (Eds.) Matices Interdisciplinarios en Estudios Fitoliticos y de otros Microfósiles, BAR (British Archaeological Reports) International Series S1870, Capitulo 9. Oxford University Press, Oxford, p. 3-21.

Zucol, A.F., Brea, M. y Bellosi, E. 2010 a. Phytoliths studies in Gran Barranca (central Patagonia, Argentina): the middle-late Eocene. En: R.H. Madden, A.A.Carlini, M.G. Vucetichy R.F. Kay, (Eds.), The Paleontology of Gran Barranca. Evolution and environmental changes through the Middle Cenozoic of Patagonia. Cambridge University Press, Cambridge, p. 317-340.

Zucol, A. F., Passeggi, E., Brea, M., Patterer, N.I., Fernández Pepi, M.G. y Colobig, M.M. 2010 b. Phytolith analyses for the Potrok Aike Lake Drilling Project: Sample treatment protocols for the PASADO Microfossil Manual. En: H. Corbella y N.I. Maidana (Eds.), 1a Reunión Internodos del Proyecto Interdisciplinario Patagonia Austral y $1^{\text {er }}$ Workshop Argentino del Proyecto Potrok Aike Maar Lake Sediment Archive Drilling Project. Proyecto Editorial PIPA (Buenos Aires), Programas y Resúmenes: 81-84.

doi: 10.5710/AMGH.16.05.2013.577

Recibido: 6 de marzo de 2012

Aceptado: 16 de mayo de 2013 